\title{
Experimental Aerodynamic Simulation of Scallop Ice Accretion on a Swept Wing
}

\author{
Brian Woodard, ${ }^{1}$ Andy Broeren, ${ }^{2}$ Mark Potapczuk, ${ }^{2}$ Sam Lee, ${ }^{3}$ Christopher Lum, ${ }^{4}$ Michael Bragg, ${ }^{4}$ and \\ Timothy Smith ${ }^{5}$ \\ ${ }^{1}$ University of Illinois \\ ${ }^{2}$ NASA Glenn Research Center \\ ${ }^{3}$ Vantage Partners Limited \\ ${ }^{4}$ University of Washington \\ ${ }^{5}$ Federal Aviation Administration Technical Center
}

\begin{abstract}
Understanding the aerodynamic impact of swept-wing ice accretions is a crucial component of the design of modern aircraft. Computersimulation tools are commonly used to approximate ice shapes, so the necessary level of detail or fidelity of those simulated ice shapes must be understood relative to high-fidelity representations of the ice. Previous tests were performed in the NASA Icing Research Tunnel to acquire high-fidelity ice shapes. From this database, full-span artificial ice shapes were designed and manufactured for both an $8.9 \%$-scale and $13.3 \%$-scale semispan wing model of the CRM65 which has been established as the full-scale baseline for this sweptwing project. These models were tested in the Walter $\mathrm{H}$. Beech wind tunnel at Wichita State University and at the ONERA F1 facility, respectively. The data collected in the Wichita St. University wind tunnel provided a low-Reynolds number baseline study while the pressurized F1 facility produced data over a wide range of Reynolds and Mach numbers with the highest Reynolds number studied being approximately $R e=11.9 \times 10^{6}$. Past work focused on only three different fidelity variations for ice shapes based on multiple icing conditions. This work presents a more detailed investigation into several fidelity representations of a single highly three-dimensional scallop ice accretion. Sensitivity to roughness size and application technique on a low-fidelity smooth ice shape is described. The data indicate that the aerodynamic performance is not especially sensitive to the grit variations. An ice accretion code was also used to generate ice shapes for aerodynamic testing and comparisons. These ice shapes have a general appearance like the low-fidelity smooth ice shapes, but in this case, the computer-generated ice shape is significantly smaller. As such, the impact of that ice shape on the aerodynamic performance of the wing is reduced compared to the smooth ice shape based on the icing experiment for those same conditions. Spanwise discontinuities were also introduced to a lowfidelity ice shape in an attempt to quantify the impact of those variation in the high-fidelity ice shape. While the lift data indicate good agreement between the high-fidelity ice shapes and the lowfidelity ice shapes with spanwise discontinuities, a closer investigation of the data suggests potential, significant differences in the flowfield. These results were similar at both facilities over the wide range of test conditions utilized.
\end{abstract}

\section{Introduction}

Understanding the effects of ice accretion on the aerodynamic performance of large scale, swept wings is a complicated problem affecting the design and certification of transport aircraft. These effects on highly three-dimensional wings are currently not well

Page 1 of 22 understood and present a significant challenge to airframe manufacturers, certification authorities, and research organizations. In an effort to understand this problem, a large collaborative research program has been sponsored by NASA, the Office National d'Etudes et Recherches Aérospatiales (ONERA), and the Federal Aviation Administration (FAA). Broeren et al.[1] describe the objectives of this project in more detail. Two of the primary goals involve creating a database of realistic publically-available, swept-wing ice accretions and performing aerodynamic assessments of the aerodynamic effects of those ice accretions. Aircraft manufacturers and regulators are increasingly interested in simulation tools for aircraft icing, but validation cases are necessary for these types of ice accretions. These validation ice shapes and aerodynamic performance measurements are provided by this project for swept wings. Extensive past research into the aerodynamic performance effects of ice accretion has primarily focused on airfoils and straight wings which are generally two-dimensional. Bragg et al.[2] reviewed this research topic and classified ice accretions by their aerodynamic effect. During the initial phase of this research program, the swept-wing icing literature was reviewed in an effort to classify these ice accretions also by aerodynamic impact $[3,4]$. With the completion of the aerodynamic wind-tunnel testing phase of the project, a review of the ice-accretion classifications is likely necessary.

Swept-wing ice accretions are extremely complex geometrically. Many icing conditions result in highly three-dimensional ice accretions that are often referred to as "scallops" or "lobster tails" due to their appearance and the substantial spanwise variations in the ice. Icing simulation tools like those currently being developed by NASA and ONERA have been applied through this research project $[5,6]$ but are unable to capture these three-dimensional variations. However, the level of fidelity to which these variations need to be captured in order to accurately predict the aerodynamics is not fully understood. One way to quantify that fidelity requirement is to test various fidelity representations of the ice shapes for their aerodynamic performance effects. As part of this collaborative research effort, an ice accretion database for large-scale swept wings has been created based on a series of tests in the Icing Research Tunnel (IRT) at the NASA Glenn Research Center [7]. One single, highly three dimensional ice shape forms the basis for the fidelity variations studied in this paper.

In aircraft icing research, ice accretions are commonly recreated to be attached to airfoils or wings for testing in dry-air wind tunnels. Generally, these recreated representations are referred to as artificial ice shapes. Throughout this work, several fidelity variations of these artificial ice shapes will be explored. A past NASA-ONERA research project investigated geometric fidelities associated with 
developing these artificial ice shapes [8]. During that project, the current methods for capturing three-dimensional geometries were not available, but more recently a method for creating high-fidelity artificial ice shapes has been validated using 3-D laser scanning and rapid-prototype manufacturing [9]. That methodology was utilized for this project. With these capabilities applied to the swept-wing ice accretion database, numerous artificial ice shapes are available for wind tunnel testing for aerodynamic performance effects. Both lowand high-Reynolds number aerodynamic testing have been performed using these ice shapes. The ice accretion database and the aerodynamic wind tunnel models are all based on the Common Research Model (CRM) [10-13]. For this project, the full-scale geometry is a $65 \%$ scale version of the CRM, designated here as CRM65 [1]. The design of the icing tunnel models is described by Fujiwara et al. in several publications [14-18]. Low-Reynolds number testing was performed using an $8.9 \%$ scale model of the CRM65, and the high-Reynolds number testing utilized a 13.3\% scale model. Broeren et al.[19], Camello et al.[20], Lum et al.[21], and Sandu et al.[22] have presented results related to the lowReynolds number aerodynamic testing with Camello et al. making a preliminary investigation into the ice shape fidelity effects relating to the aerodynamics. High-Reynolds number results from this project have previously been reported by Broeren et al.[23], Lee et al.[24], and Woodard et al.[25] showing results from ice shape fidelity studies over a range of Reynolds and Mach numbers and comparisons between the two different facilities.

This paper will present the results of several fidelity-variation studies on a highly three-dimensional scallop ice shape that has been tested at low- and high-Reynolds number. The primary data used for these comparisons come from the force balance measuring the integrated performance of the wing. Additional data such as model surface pressures and flow visualization techniques (minitufts and surface-oil flow) were collected for many configurations and can provide further insight regarding the flowfield. However, that analysis is beyond the scope of the current work. Comparisons are made between lift- and drag-based performance parameters over a large range of Reynolds numbers representing two different scale wind tunnel models tested at different facilities. Companion papers by Broeren et al.[26] describe the results from fidelity variations of a glaze ice accretion, and Lee et al.[27] provide a detailed comparison of the results between the two facilities utilized throughout this project. This paper summarizes a roughness sensitivity study, a comparison between computer- and experimentally-generated ice shapes, and a preliminary investigation into the creation of highly three dimensional ice shapes from smooth ice shapes by introducing spanwise discontinuities in the ice shape.

\section{Wind-Tunnel Facility, Model, and Experimental Methods}

\section{A. Wind-Tunnel Facilities}

The majority of the experimental data described in this work were collected at the ONERA F1 pressurized wind tunnel located at the Fauga-Mauzac Center in southern France. The closed-return tunnel can be pressurized to $56 \mathrm{psi}$ and has a test section approximately 11.5-ft high x 14.8-ft wide. The pressurization capability provides independent Reynolds and Mach number control over a range of $R e=$ $1.6 \times 10^{6}$ to $11.9 \times 10^{6}$ and $M=0.09$ to 0.34 for the tests described. Not all possible combinations of Reynolds and Mach number in that range are achievable in the facility simultaneously. Broeren et al.[23] describe the complete test matrix and other details of the facility in more depth, so only the basic description of the facility for understanding the tests is provided here. A maximum of thirteen combinations of Reynolds and Mach number were utilized for testing various icing configurations. For some configurations, conditions were omitted in order to optimize the limited test time in the wind tunnel. Angle of attack sweeps were performed with a continuous change in pitch angle at a constant rate of $0.1 \mathrm{deg} / \mathrm{sec}$. The model angle of attack was varied from -6 deg up to 25 deg except in cases where dynamic forces limited the maximum angle of attack or a clear local maximum in lift coefficient was measured. During each of those angle of attack sweeps, the aerodynamic forces were measured along with the surface pressures. Broeren et al.[23] also describe the data acquisition system in greater detail including the load ranges and associated uncertainty for each of calculated aerodynamic performance parameters. The normal and axial force values are measured using an external balance below the floor of wind tunnel that turns with the model as the angle of attack changes. The lift and drag are then calculated in the usual way to determine the forces in the coordinate system based relative to the free-stream velocity.

Since the data were collected as the model rotated continuously about the pitch axis, a custom post-processing routine was developed in order to conditionally average the continuous data into discrete data points. The plots in the paper utilize this post-processed data with 0.5 deg. angle of attack increments. The pitching-moment coefficient was referenced to the quarter-cord of the mean aerodynamic chord. Previously published work for this swept-wing icing research project referenced the pitching-moment coefficient to a different location on the wing. In particular several papers and reports $[19,20,28]$ summarizing lower-Reynolds number tests with the same model geometry used a different reference location.

The lower Reynolds number wind-tunnel tests were performed at the Walter H. Beech Memorial Wind Tunnel on the campus of Wichita State University (WSU). This closed-return wind tunnel operates at atmospheric pressure, so Reynolds number and Mach number aerodynamic effects cannot be independently investigated. The test section measures 7 -ft high $\times 10$-ft wide. The data presented were measured at approximately $R e=1.6 \times 10^{6}$ and $M=0.17$ or $R e=$ $2.4 \times 10^{6}$ and $M=0.26$. The details of the experimental setup along with an uncertainty analysis can be found in Woodard et al.[28] and the initial wind tunnel tests are described by Broeren et al.[19] and Camello et al.[20]. The data from the force balance and the surface pressures was acquired in a standard pitch-pause method, so postprocessing the data into discrete points was not necessary.

All aerodynamic data $\left(\alpha, C_{L}, C_{M}, C_{D}\right.$ and $\left.C_{p}\right)$ presented in this paper were corrected for wind-tunnel-wall effects. The data from the F1 facility are corrected using an in-house ONERA method that yields two corrections terms, one of which is constant based on the model and wind tunnel geometry and the other proportional to the model lift coefficient. The data collected in the lower-Reynolds number, atmospheric-pressure wind tunnel at WSU are corrected using the procedure for 3D model corrections outlined in Barlow et al.[29] and implemented by WSU [30]. The magnitude of the correction to the angle of attack applied in the WSU facility has been reduced relative to past papers and reports related to this project $[19,20,28]$. Lee et al.[24] describe the motivation for the change to the angle of attack correction. In both facilities, the corrections are performed in realtime as part of the data acquisition process. Direct comparisons of aerodynamic performance parameters from the two facilities are avoided in this paper, and only relative effects are presented. However, the companion paper by Lee et al.[27] provides direct 
comparison and analysis of the results from the two different facilities.

\section{B. Wind-Tunnel Models Description}

The two semispan models fabricated for these wind-tunnel tests were based on an $8.9 \%$ and $13.3 \%$ scale version of the CRM65 wing. Due to the facilities for which the models were designed, the $8.9 \%$ scale model is referred to as the WSU model, and the $13.3 \%$-scale model is referred to as the F1 model. Table 1 summarizes the geometric parameters of the wings. The F1 model is exactly $50 \%$ larger than the WSU model. The full-scale, original CRM geometry has a realistic cruise configuration loading applied to the wing resulting in a wing shear similar to dihedral [13]. In order to simplify the design of the removable leading edge segments (described below), this shearing or "bending" of the wing was removed from the model geometry resulting in an unsheared wing with a straight leading edge across the span of the model. The wing retains the twist and taper of the original CRM. A planform view of the F1 model is shown in Fig. 1 with key dimensions.

Table 1. Summary of 8.9\% and 13.3\% Scale CRM65 Semispan Wing Geometric Parameters.

\begin{tabular}{lll}
\hline \hline $\begin{array}{l}\text { Wing } \\
\text { Parameter }\end{array}$ & $\begin{array}{l}13.3 \% \text { Scale } \\
(\text { F1 Model) }\end{array}$ & $\begin{array}{l}8.9 \% \text { Scale } \\
\text { (WSU Model) }\end{array}$ \\
\hline Span, $b$ & $7.5 \mathrm{ft}(90.00 \mathrm{in})$ & $5.0 \mathrm{ft}(60.00 \mathrm{in})$ \\
$M A C$ & $2.08 \mathrm{ft}(25.01 \mathrm{in})$ & $1.39 \mathrm{ft}(16.67 \mathrm{in})$ \\
Area (Geometric) & $13.55 \mathrm{ft}^{2}\left(1951.0 \mathrm{in}^{2}\right)$ & $6.01 \mathrm{ft}^{2}\left(865.3 \mathrm{in}^{2}\right)$ \\
Volume & $2.09 \mathrm{ft}^{3}\left(3604.5 \mathrm{in}^{3}\right)$ & $0.617 \mathrm{ft}^{3}\left(1069 \mathrm{in}^{3}\right)$ \\
Aspect ratio $\dagger$ & 8.3 & 8.3 \\
Taper ratio & 0.23 & 0.23 \\
Root chord & $3.38 \mathrm{ft}(40.50 \mathrm{in})$ & $2.25 \mathrm{ft}(27.00 \mathrm{in})$ \\
Tip chord & $0.77 \mathrm{ft}(9.28 \mathrm{in})$ & $0.52 \mathrm{ft}(6.19 \mathrm{in})$ \\
Root $\alpha$ & $4.4 \mathrm{deg}$. & $4.4 \mathrm{deg}$. \\
Tip $\alpha$ & $-3.8 \mathrm{deg}$. & $-3.8 \mathrm{deg}$. \\
$1 / 4$-chord sweep angle & $35 \mathrm{deg}$. & $35 \mathrm{deg}$. \\
Leading edge sweep angle & $37.2 \mathrm{deg}$. & $37.2 \mathrm{deg}$. \\
Location of rotation center $\ddagger$ & $x=29.05 \mathrm{in} ., z=0$ & $x=19.37 \mathrm{in} ., z=0$ \\
Location of moment center $\neq$ & $x=35.80 \mathrm{in} ., z=0$ & $x=23.87 \mathrm{in} ., z=0$ \\
Location of $0.25 \times M A C \ddagger$ & $x=26.23 \mathrm{in} ., z=0$ & $x=17.49 \mathrm{in} ., z=0$ \\
\hline \hline
\end{tabular}

$\dagger$--While the other parameters in this table are defined specifically for this model, the aspect ratio is defined for a complete airplane configuration using the formula, $\frac{(2 \times \text { semispan })^{2}}{2 x \text { area of one wing }}$.

$\ddagger-(0,0,0)$ is the wing root-section leading edge at zero angle of attack.

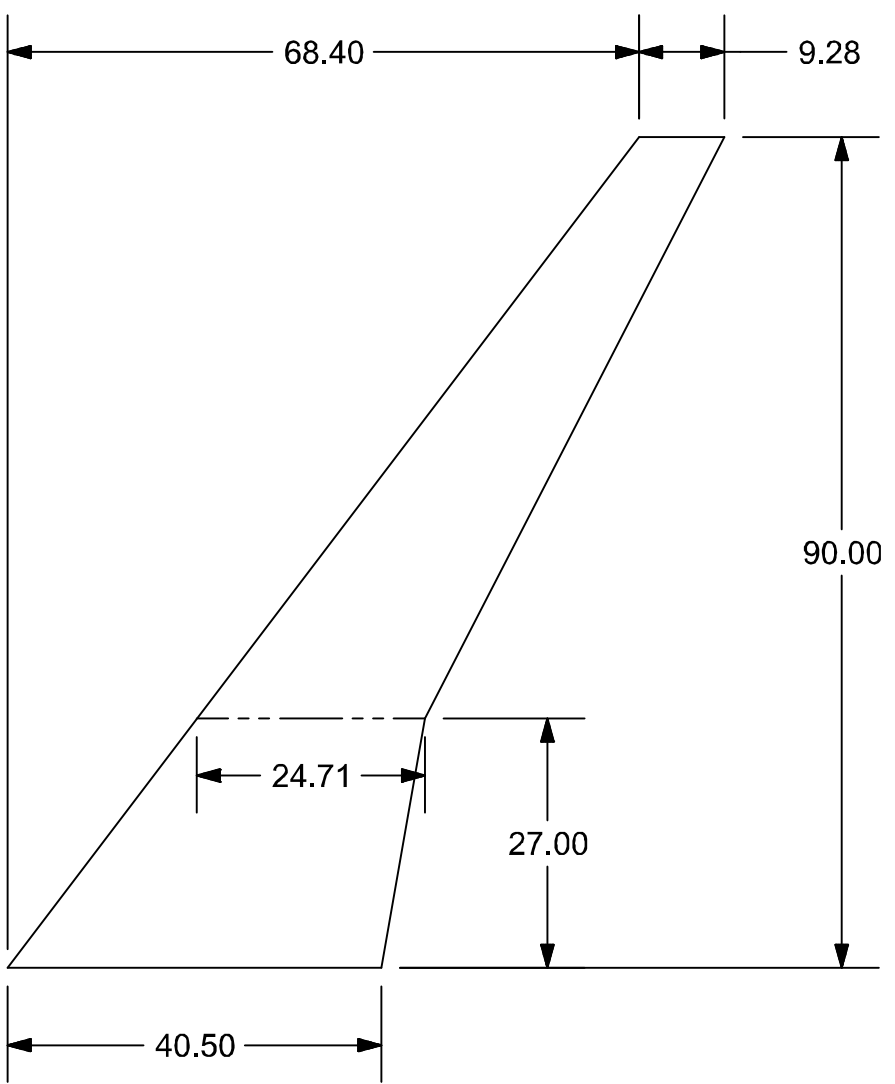

Figure 1. 13.3\% scale (F1 Model) CRM65 semispan wing planform with key dimensions labeled in inches.

For the F1 model, the main body was machined from stainless steel while the removable leading edge components were machined from aluminum. For the WSU model, both the main element and the removable leading edge components were aluminum. The F1 model contained 243 pressure taps in its clean configuration, while the WSU model contained 219. Figure 2 shows photographs of the wing models installed in their respective wind tunnels with circular splitter plates. An artificial ice shape is mounted to the leading edge of the F1 model, and the WSU model is shown in the clean configuration. Below the circular splitter plate shown in the images, a streamlined shroud isolates the wing spar from any aerodynamic loads. With this arrangement, both the splitter plate and shroud were non-metric meaning the aerodynamic forces were only measured on the wing itself. The designs of the splitter plate and shroud were specifically investigated during a preliminary test campaign utilizing the WSU model prior to the design and fabrication of the F1 model $[19,28]$. 

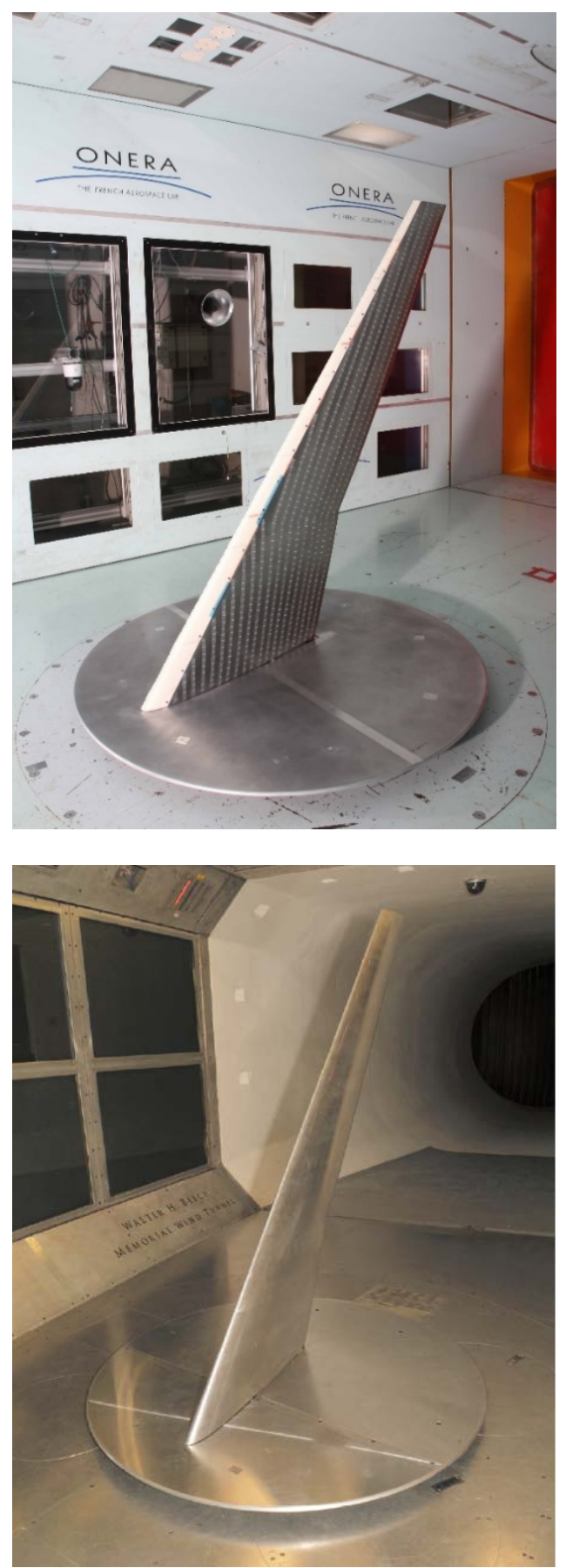

Figure 2. Photographs upper surface of subscale CRM65 semispan wing models installed in their respective wind tunnels; left image shows 13.3\% scale CRM65 semispan wing installed in ONERA F1 test section; right image shows $8.9 \%$ scale CRM65 semispan wing installed in WSU test section.

The model was designed and built with a removable leading edge that allowed artificial ice-shapes to be added to the wing. This approach has been used in previous icing aerodynamic studies [8,31,32,33,34] and allows for efficient and repeatable changes of the artificial iceshape configurations. The efficiency was particularly important during this research effort when a large number of ice-shape configurations were investigated in limited test time. The main components of each model were: the main element (including a spar that attached to the force balance); a full-span clean leading edge; and a partial-span leading edge used for mounting ice shapes. An open channel exists between the main element and any of the leading edge components for routing pressure tubing out through the base of the model to the data acquisition system. The partial-span removable leading edge extended from the root to $83 \%$ of the semispan for the F1 model and from the root to $50 \%$ of the semispan for the WSU model. Both contained a portion of the airfoil contour on the lower surface. Artificial ice shapes were attached to these removable leading edges and covered the entire upper surface of this removable leading edge. Outboard of this partial-span leading edge, the artificial ice shapes were attached directly to the main element. The model is too thin at the outboard portion of the wing to support the addition of a full-span removable leading edge. This design does not adversely affect the efficiency or repeatability of the artificial iceshape configuration changes.

The artificial ice shapes were created using a rapid prototype manufacturing (RPM) technique called stereo-lithography (SLA). The SLA process utilizes an ultraviolet laser to solidify liquid polymer resins. The majority of the artificial ice shapes were manufactured from the Somos NeXt brand polymer. Some of the early ice shapes were rapid prototyped using Accura 60. The tolerances are advertised to be about $+/-0.005$ inches for this process for either polymer. The Somos NeXt material was selected due to desirable advertised properties associated with the stability of the printed parts. The project potentially requires artificial ice shapes to be in storage for over one year between tests. Early work with SLA products proved challenging with regard to long-term storage. Mitigating exposure to water vapor in the air and ultra-violet radiation has been shown to drastically improve the lifetime of the parts for repeat testing. The process for creating an artificial ice shape involves adding the full-span ice shape to the necessary wing geometry, and then dividing the ice shape into sections. The leading edge was divided into three segments for the F1 model and two segments for the WSU model. All segments were approximately 37.5 inches long. Pressure taps were installed in each of these segments at the same locations as on the clean removable leading edge. The pressure tap holes were included in the RPM design, and then stainless steel tubes were glued into each hole and plumbed to a quick disconnect inside the channel between the removable leading edge and the main element.

The pressure taps in the models were primarily distributed in streamwise rows across the span of the model (Fig. 3). The taps in the main element of the model were plumbed with stainless steel tubing from their location on the surface out the root of the model. The routing of the taps in the removable leading edges required a more complicated design. The stainless steel tubing in both the clean leading edge and in the RPM ice leading edges transitioned to plastic tubing and then connected to a Scanivalve quick disconnect fitting. The use of these fittings allowed relatively quick model reconfigurations between clean and various RPM leading edges. 


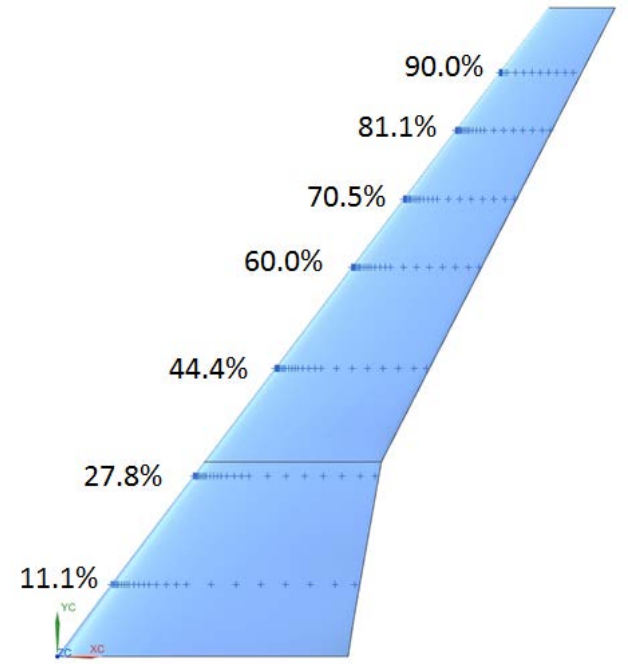

Figure 3. Pressure tap locations on upper surface of 13.3\% scale CRM65 (F1 model).

\section{Artificial Ice-Shape Configurations}

Throughout this project, many artificial ice-shape configurations have been designed and tested. Camello et al.[20] summarized the ice shapes that have been tested at WSU. These artificial ice shapes include high-fidelity representations based directly on icing wind tunnel testing and various lower fidelity representations of those high-fidelity ice shapes or their associated icing conditions. Table 2 shows the conditions in the IRT resulting in the highly threedimensional ice accretion, Maximum Scallop, and the flight reference conditions to which these conditions correspond. These flight reference conditions are important for simulations that are described later in this section. From the ice shapes resulting from these conditions, many different fidelity representations have been designed, built, and tested in either the F1 or WSU facility.

Table 2. Summary of icing conditions resulting in highly three-dimensional ice accretion.

\begin{tabular}{lcc}
\hline \hline Icing Conditions & $\begin{array}{c}\text { Maximum Scallop } \\
(\text { IRT })\end{array}$ & $\begin{array}{c}\text { Flight Reference } \\
\text { Condition }\end{array}$ \\
\hline Angle of Attack (deg) & 3.7 & 3.7 \\
Speed (kts) & 130 & 232 \\
Mach Number & 0.20 & 0.36 \\
Total Temperature $\left({ }^{\circ} \mathrm{C}\right)$ & -6.3 & -3.0 \\
Static Temperature $\left({ }^{\circ} \mathrm{C}\right)$ & -8.5 & -10.0 \\
Cloud MVD $(\mu \mathrm{m})$ & 25 & 20 \\
Cloud LWC $\left(\mathrm{g} / \mathrm{m}^{3}\right)$ & 1.0 & 0.55 \\
Exposure Time $(\mathrm{min})$ & 29 & 29.3 \\
\hline
\end{tabular}

Ice-accretion testing was performed in the NASA Icing Research Tunnel using three individual CRM65 section models with full-scale leading edges. Details of the icing tests associated with this project are provided by Broeren et al.[7]. The three sections, referred to as the Inboard, Midspan, and Outboard, represent $y / b$ of $0.20,0.64$, and 0.83 , respectively. Icing tests were performed for each of the models at the conditions listed in Table 2, and the resulting ice shapes were captured using a 3D laser scanning method [9]. Full-span ice shapes were then developed from the three laser-scanned sections. Camello et al.[35] describe the process by which the ice is interpolated and extrapolated to cover the entire leading edge of the wing. The specific conditions for the icing tests were nominally based on holding conditions for a CRM65 aircraft in the Code of Federal Regulations, Part 25, Appendix C, Continuous Maximum. The particular conditions resulting in the Maximum Scallop ice shape resulted from a temperature sweep while holding other conditions constant. The temperature sweep started within the Appendix C envelope, but the Maximum Scallop conditions are outside of Appendix C. Images of the actual ice shapes from the three IRT test models are shown in Fig. 4 for the icing conditions described by Table 2. The names of the ice shapes are based on the general appearance of the ice although all of the shapes show significant spanwise variation. The "Maximum Scallop" ice shape has welldefined scallops or lobster tails at all three spanwise stations. As later images will illustrate, the Maximum Scallop ice shape is a large accretion, and the highly three-dimensional features dominate the entire span.
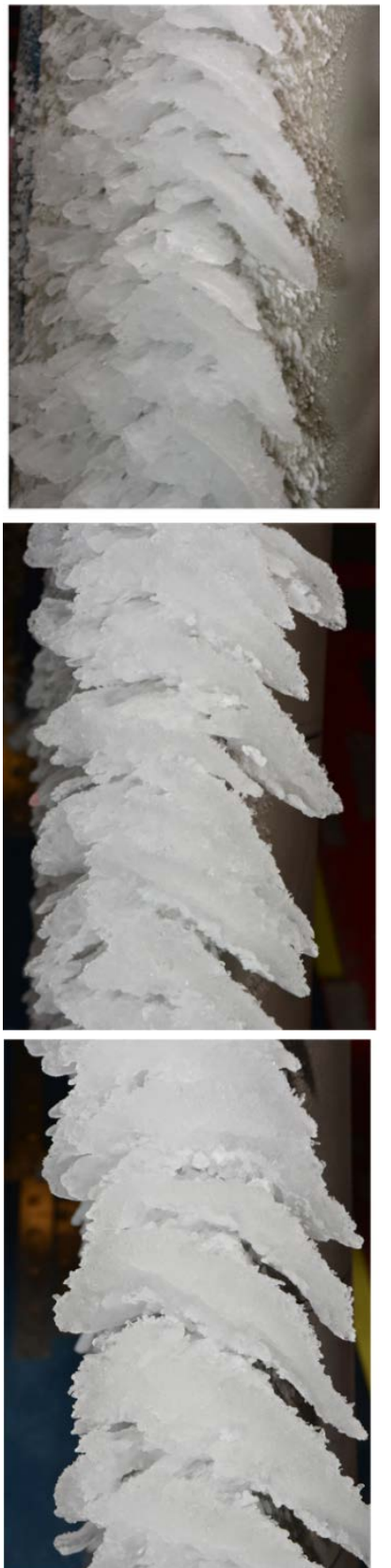

Figure 4. Photographs of Maximum Scallop ice accretion on Inboard (top), Midspan (middle), and Outboard (bottom) models.

Page 5 of 22 
While the images shown in Fig. 4 represent the three dimensionality of the ice shapes well, they do not easily illustrate the size of the ice. Further comparisons are shown in Fig. 5 where the maximum combined cross section (MCCS) is plotted for each of the spanwise locations. The MCCS was derived from 30 section cuts over a sixinch spanwise segment of the 3D ice scan [7]. The section cuts were projected onto a single plane and the maximum outer boundary was obtained. The resulting MCCS profile represents the outermost extent of the ice shape over that six-inch segment. The three dimensionality of the ice shapes is not obvious in these MCCS plots, but the size variations are evident.
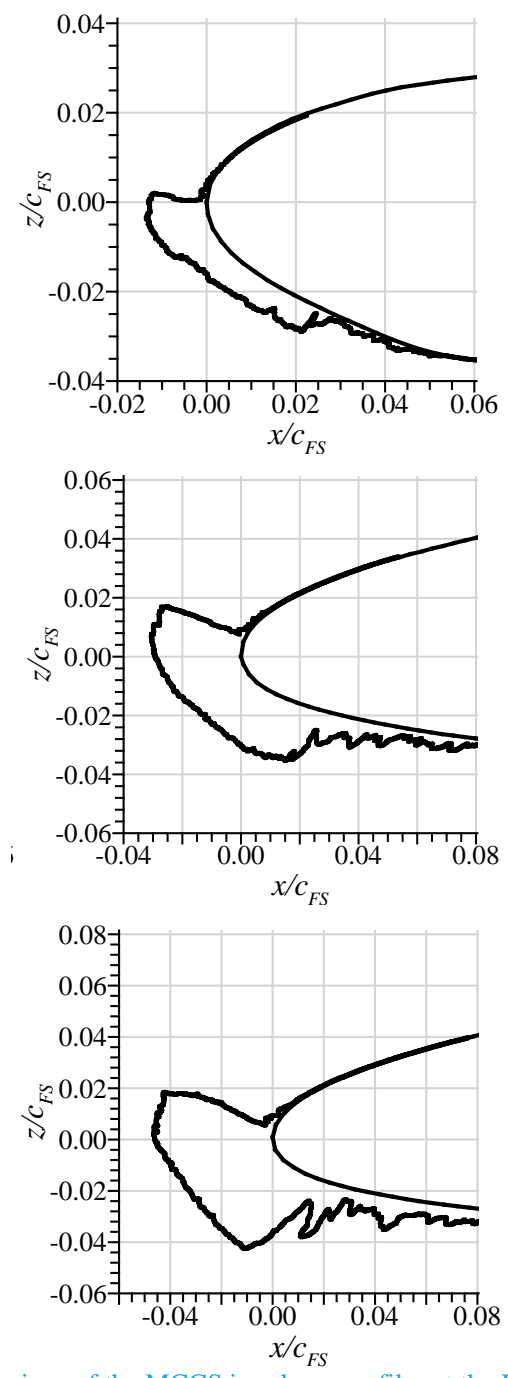

Figure 5. Comparison of the MCCS ice-shape profiles at the Inboard (top), Midspan (middle), and Outboard (bottom) sections.

The full-span ice shapes that are developed from the laser scans contain all of the highly three-dimensional properties of the original ice shape. In terms of fidelity, these ice shapes are called "high fidelity" meaning that they are the most detailed ice shapes available based on current understanding and technology. The limitations of the current method for actual reproduction of the ice shapes are described by Camello et al.[35] as they describe the details of the process by which the laser scans of the ice shapes are transformed into the wind-tunnel model ice shapes. Various lower fidelity representations can also be created based on the full-span highfidelity shapes. The ice shapes described as “3D Smooth” are built Page 6 of 22 by taking section cuts along the span, smoothing these cuts, and then lofting them to build a new, full-span ice shape. An example comparison between a high fidelity and 3D smooth ice shape is shown in Fig. 6. The figure only includes a small segment of the span that is representative of the entire ice shape.

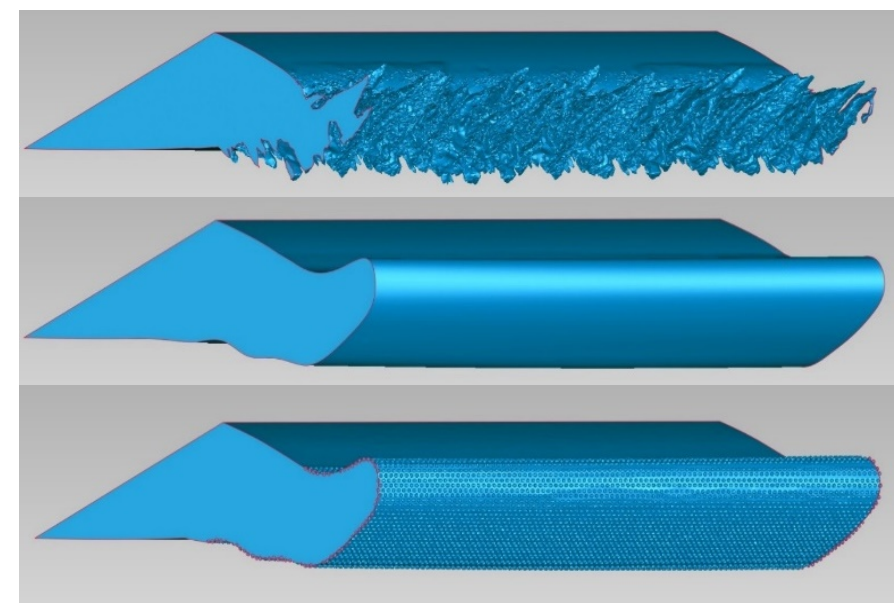

Figure 6. Comparison of high-fidelity artificial ice shape geometry (top) with the lower-fidelity, 3D smooth geometry (middle) and 3D smooth with hemispherical roughness (bottom).

These 3D smooth ice shapes are spanwise smooth in the sense that no scallops are represented, but they are three dimensional such that the ice horn height and angle vary across the span. Some past publications have described these lower-fidelity ice shapes as "2D Smooth," but that naming convention implies that the cross section of the ice shape does not vary across the span. The ice shapes detailed in this work and described as 3D smooth have substantial spanwise variation as they are derived directly from the high-fidelity ice shapes. They simply do not have the scallop features present in the high-fidelity ice shapes. An additional category of lower-fidelity ice shapes, named “3D Simple” ice shapes, has been developed to investigate the aerodynamic impact of specific ice-shape features, but they are not part of this specific work. An investigation into the effects of those ice shapes, based on different icing conditions compared to this work, can be found in Broeren et al.[26].

Another, intermediate fidelity between the high-fidelity and 3D smooth ice shapes involves adding grit roughness to the 3D smooth ice shapes in order to simulate some ice roughness. The recommended roughness size of $3 \mathrm{~mm}$ for the full-scale aircraft comes from FAA Advisory Circular 25-25A [36]. This corresponds to approximately 46 grit size on the $13.3 \%$ model and 60 grit size on the $8.9 \%$ model. The grit is adhered using epoxy and is applied with an extremely dense coverage. In order to better understand the aerodynamic sensitivity to the roughness size, 3D smooth ice shapes were tested in both facilities with grit corresponding to both half and double the size recommended by the FAA Advisory Circular. The exact size of the grit utilized during the tests is dictated by the sizes of commercially available silicon carbide grit. Table 3 shows the target sizes based on scaling the FAA recommendation to the size of the model and the actual grit size used. 


\begin{tabular}{cccc}
\hline \hline & & \multicolumn{2}{c}{ Model } \\
\cline { 3 - 4 } & & High $R e$, & Low $R e$, \\
& $13.3 \%$ Scale & $8.9 \%$ Scale \\
\hline Standard & Target Height (inches) & 0.0157 & 0.0105 \\
Size & Grit Size Utilized & 46 & 60 \\
\hline Half & Target Height (inches) & 0.0079 & 0.0053 \\
Size & Grit Size Utilized & 80 & 100 \\
\hline Double & Target Height (inches) & 0.0314 & 0.0210 \\
Size & Grit Size Utilized & 20 & 36 \\
\hline Hemisphere & Height (inches) & 0.0150 & 0.0100 \\
\hline \hline
\end{tabular}

In addition to the standard grit adhered to the 3D smooth ice shapes, hemispherical roughness was also tested. These ice shapes were designed by patterning hemispheres over the entire surface of the 3D smooth ice shape. The height (radius) of the hemispheres was scaled to match the size recommended in the FAA Advisory Circular, but the coverage density does not match those guidelines. Those heights are included in Table 3. The nature of hemispherical geometry does not allow both the recommended height and density to be simultaneously satisfied. Increasing the density sufficiently would result in overlapping hemispheres defeating the height from the surface requirement. The hemispheres were designed such that the center of each hemisphere is 1.3 times the diameter from each neighboring hemisphere. The bottom image in Fig. 6 illustrates the hemispherical roughness added to the 3D smooth ice shape. Images of three of the fidelity configurations installed on the F1 model are shown in Fig. 7. The three dimensionality of the high fidelity artificial ice shape is evident in the images. The ice shapes with grit roughness added to them illustrate that the roughness was applied over the entire ice shape and that it was applied with high density.

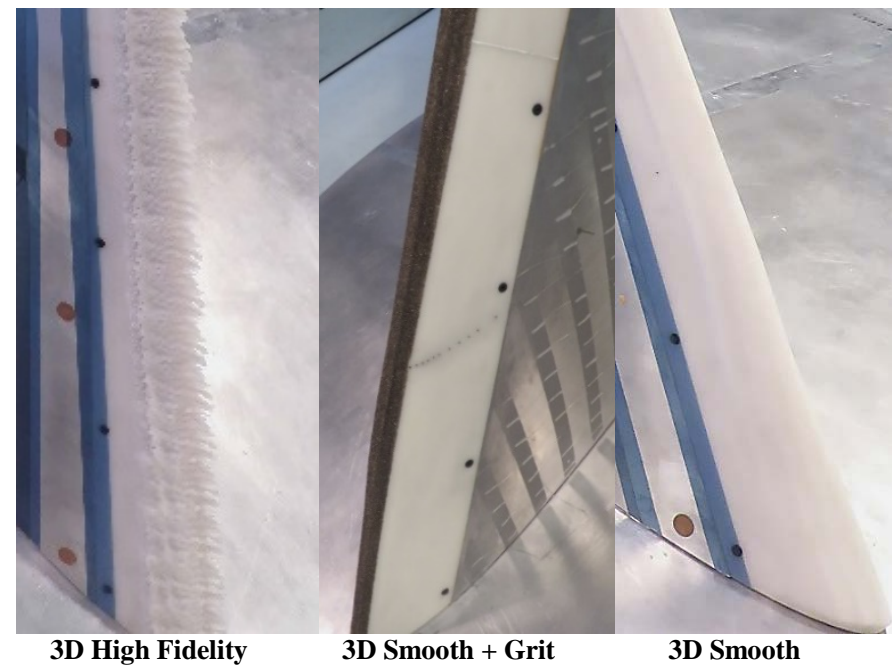

Figure 7. Images showing three of the fidelity variations of the Max Scallop ice shape installed on the wind-tunnel model.

Ice shapes for aerodynamic testing can also be created from computer simulations. Two additional ice shapes were developed based entirely on LEWICE3D simulations of the Maximum Scallop icing conditions. These ice shapes are denoted by the names LEWICE3D IRT and LEWICE3D IFB. As the name implies, the IRT-based simulations are exactly based on the geometry and conditions in the IRT. The IFB or Iced Flight Baseline simulation is based on the entire CRM65 aircraft in flight with corresponding geometry and conditions. The icing conditions are summarized in Table 2 for the
IRT test conditions and the reference conditions. They are related to each other through icing scaling best practices as described by Broeren et al.[7], but clearly, the conditions are not identical. Notably, the pressure at the flight reference case altitude is significantly lower than the atmospheric pressure in the IRT utilized in those simulations. Furthermore, the simulation geometry is not identical. The IRT simulations incorporate the models in the IRT. Fujiwara et al. [14-18] describe the design of these models in detail in several publications, and Broeren et al.[7] explain the experimental setup in the IRT. The IRT models have full-scale leading edges that match a single slice from the CRM65. The sweep of the IRT models matches the leading-edge sweep of the CRM65, and as described above, three models were used to capture the spanwise variations in the resulting ice shapes. Yadlin et al.[37] describe the computational approach whereby a 3D RANS simulation was performed using OVERFLOW at the appropriate conditions for the particular configuration. For the LEWICE3D IFB simulations, the flow solution was then used as input for the LEWICE3D simulation that was performed using a 7-bin drop size distribution and the ice density set to $450 \mathrm{~kg} / \mathrm{m}^{3}$. This analysis used the Boeing version of LEWICE3D incorporating various best practices within the Boeing Company. For the IFB, the CRM65 wing body airplane configuration was simulated which resulted in a total of 48 ice shape profiles generated along the span of the wing. These cuts were then lofted into a solid ice shape used to manufacture the corresponding artificial ice shape for aerodynamic testing. Using the same general methodology, full-span ice shapes were generated based on simulations of the IRT models. This LEWICE3D analysis was somewhat different than the one employed by Yadlin et al.[37], and the details are explained in Fujiwara et al.[38]. These simulations were performed using the NASA version of LEWICE3D, specifically the parallel version of the code called TRAJMC3D (version 2.48). A 10-bin droplet size distribution was utilized, and the ice density was set to $350 \mathrm{~kg} / \mathrm{m}^{3}$. Beyond the geometric differences between the models in the simulations, two other factors play roles in the different cross sections shown in Fig. 8 that result from the simulations. The versions of the code are not the same, and the ice density was set to a different value. As such, the IRT simulation-based ice shapes are generally larger, and especially outboard, have a lower ice-shape horn angle. A specific comparison of the cross-sectional profile of the ice shapes is shown in Fig. 8 along with the MCCS from the laser scan at each model station. For the largest section of the wing (inboard), the two LEWICE3D-based ice shapes appear similar to each other but are significantly smaller than the experimentally-based ice shape. All three of the ice shapes have a small ice horn angle relative to the oncoming flow. At the midspan location, the LEWICE3D-based shapes have a similar horn angle but are substantially different in size relative to each other. The horn angle of the experimental ice shape is significantly higher than the ones based on LEWICE3D. At the outboard station, the ice shapes all have different characteristics. The LEWICE3D IRT ice shape has a low horn angle, similar to its corresponding midspan ice shape. The LEWICE3D IFB ice shape has an ice horn angle similar to the MCCS ice shape but remains significantly smaller which is consistent with the other two stations. The LEWICE3D-based ice shapes are overall smaller at all three stations relative to the MCCS of the experimental ice shape.

The results from the LEWICE3D IRT simulations only provide an ice shape cross section at each of the three spanwise stations. A process similar to the methodology used for developing full-span ice shapes from the laser scanned data [35] was then employed to build the fullspan ice shapes. For both of these sources for the ice shapes, the fullspan ice shapes are then added to the RLE for whichever aerodynamic wind tunnel model is desired. Both methods for 
generating these ice shapes result in spanwise variations, but the variations are smooth relative to the high-fidelity ice shape. Generally, both the LEWICE3D IFB and LEWICE3D IRT ice shapes appear like the 3D smooth ice shape depicted in Figs. 6 and 7.
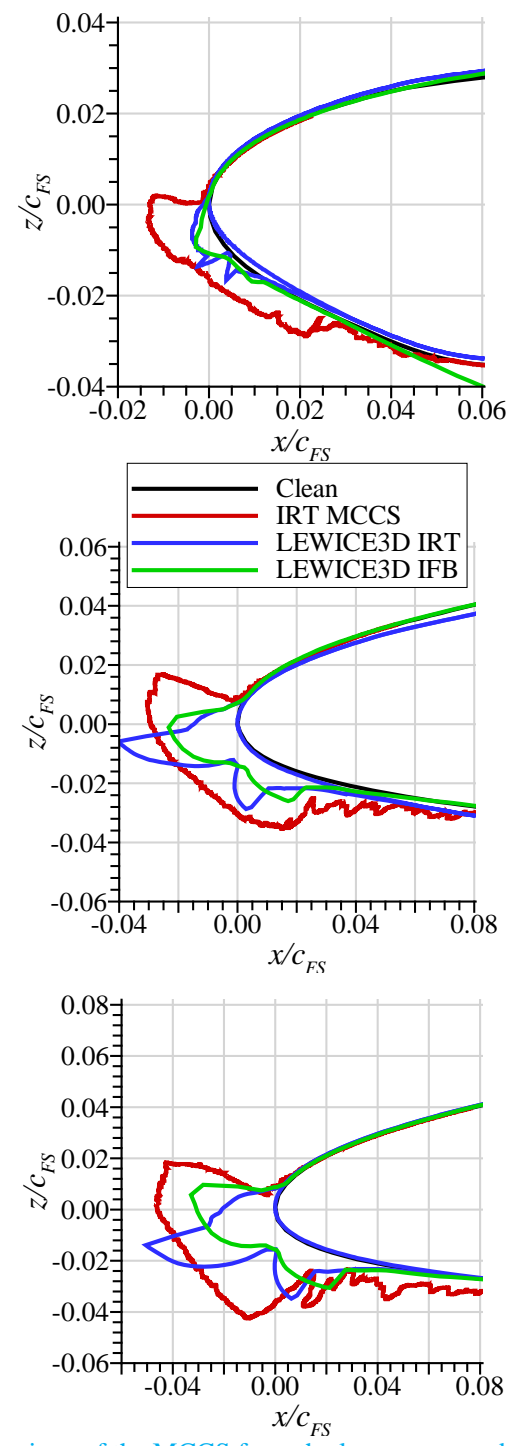

Figure 8. Comparison of the MCCS from the laser scans and two LEWICE3D-based simulations at the Inboard (top), Midspan (middle), and Outboard (bottom) sections.

The 3D smooth ice shapes drastically reduce the complexity of the ice shape by creating a shape without any of the spanwise features present in the highly three-dimensional representation. In order to investigate the aerodynamic sensitivity to the spanwise features, another fidelity of artificial ice shapes were developed. These ice shapes are referred to as "artificial scallops" as they somewhat recreate the scallops or lobster tails evident in the actual ice accretion. The concept for these ice shapes is straightforward in that material is simply removed from the 3D smooth ice shape resulting in a spanwise pattern as shown in Fig. 9. While conceptually easy to remove material from one ice shape to build another one, the details of designing these ice shapes are complicated. Since the ice shape is three dimensional on a swept wing, the orientation of the cutting plane used to create the artificial scallops could be defined in numerous ways. Additionally, the width of the scallops, size of the voids between scallops, shape of the scallops, and depth of the

Page 8 of 22 scallops relative to the clean wing could all vary across the span in a nonlinear manner. In this work, a small number of those possible variations were explored. In all of these artificial scallop ice shapes, the gaps in the ice are cut down to the clean wing, and pattern of cuts does not vary across the span with the exception of one configuration. The wing segments in Fig. 9 illustrate the 3D smooth ice shape from which the other ice shapes are based, and three different scallopspacing options.

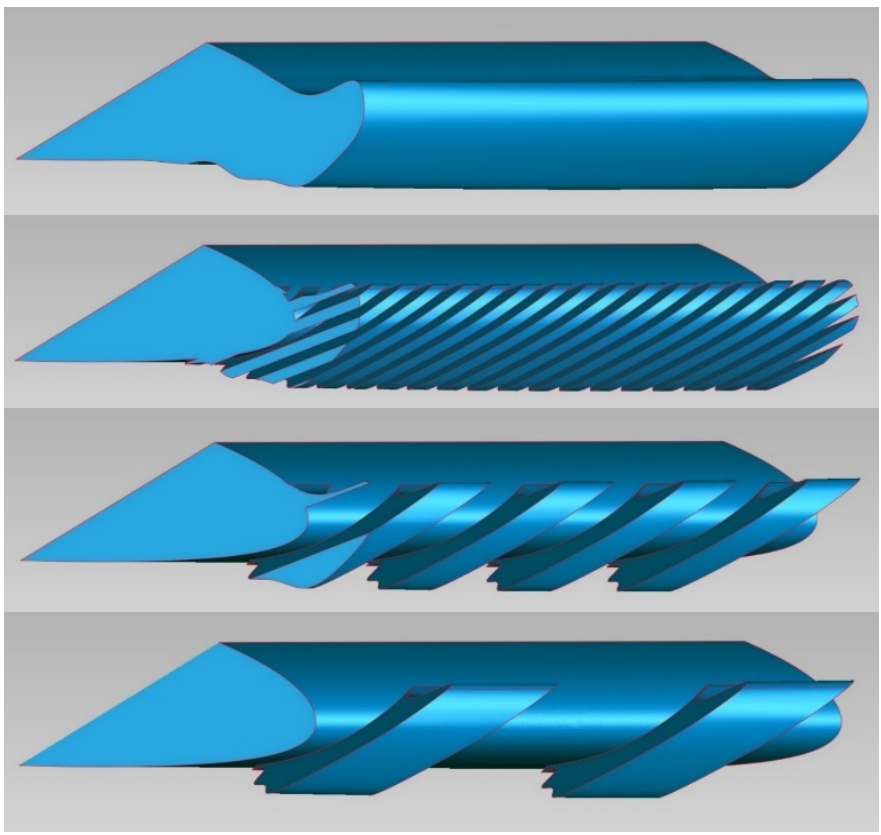

Figure 9. Models of artificial scallop ice shapes. From top to bottom, the ice shapes are: 3D smooth, Small Gap Artificial Scallop, Medium Gap Artificial Scallop, and Large Gap Artificial Scallop.

The small gap artificial scallop ice shape is the only ice shape in this category for which the scallop width and gap parameters vary across the span. Measurements were made from the full-span, high-fidelity ice shape to quantify the width of the scallop features and the gaps between them. Figure 10 shows those measurements across the span using the data markers and the curve fit that was used to manufacture the ice shape. The size of the artificial scallops utilized on the other two ice shapes illustrated in Fig. 8 are also shown in Fig. 9 for comparison to the measurements. The size of the features is scaled to the CRM65. As the figure shows, while the features do vary in size spanwise for the small gap ice shape, those variations are small compared to the size variations between the other two ice shapes. The scallops and the voids between them are approximately twice as large in each step of the progression from small to medium to large gap artificial scallop ice shape. 

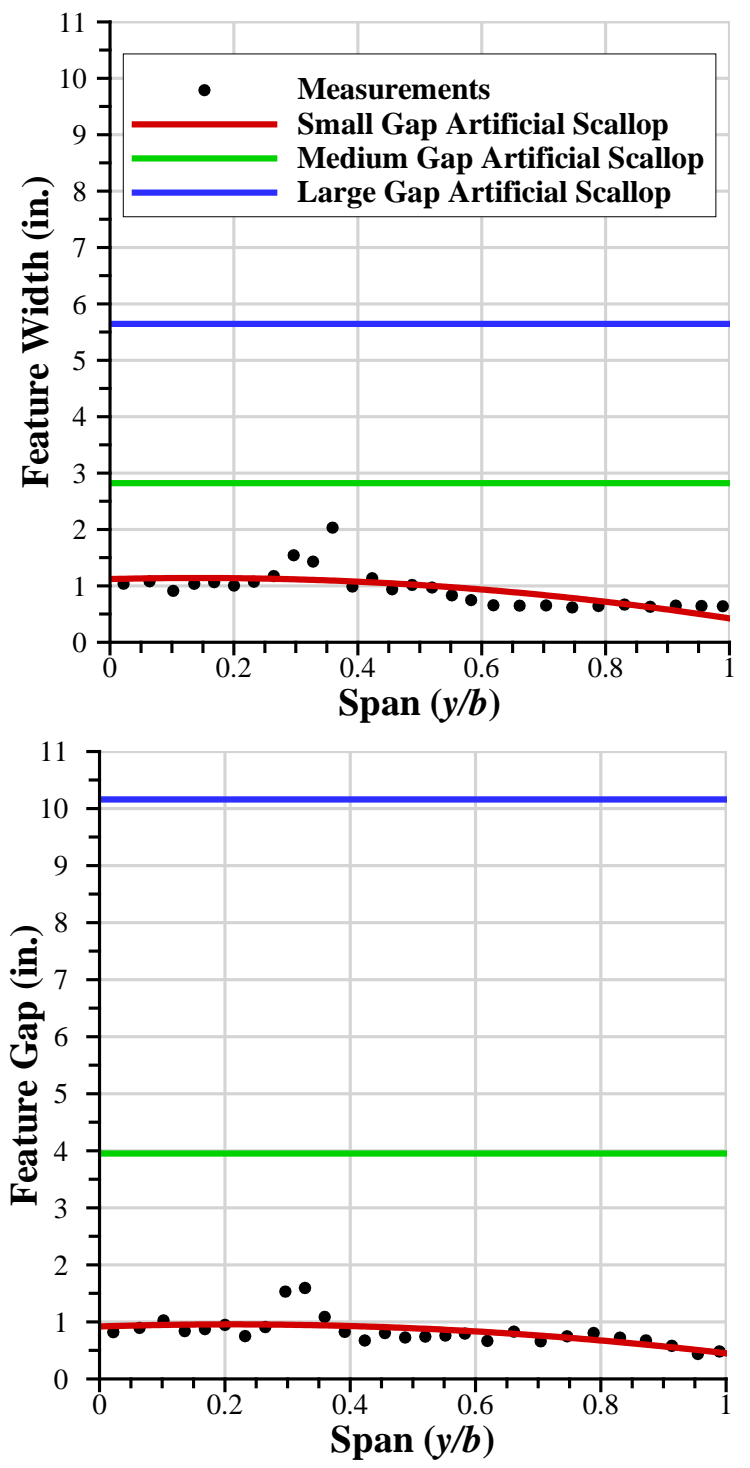

Figure 10. Spanwise variation of the size of the artificial scallop features for the three ice shapes pictured in Fig. 9. The feature size is scaled to the CRM65 (full-scale reference). Measurements from the high fidelity ice shape are also shown for reference.

The angle of the cutting plane for the gaps between the scallops can also be changed. For the three ice shapes shown in Fig. 9, the angle of that plane was chosen to approximate the angle of the scallops across the span in the high fidelity ice shape. An investigation of that angle in the full-span ice shape indicated that it does not vary significantly spanwise. In order to investigate the sensitivity of that angle, the cutting-plane angle was changed in order to develop several additional ice shapes using the medium gap artificial scallop as a baseline geometry. These ice shapes are shown in Fig. 11 with the medium gap ice shape from comparison. The angle of the cutting plane was held constant across the span, and the features and gaps between them have the same, constant size as the medium gap ice shape. The "streamwise" artificial scallop ice shape was created with the cutting plane parallel to the freestream flow. As the name implies, the "normal-to-the-leading-edge" artificial scallop ice shape was created with a cutting plane oriented normal to the leading edge of the wing or at 37.2 deg. from the freestream. The "curved" artificial scallop is generated from the normal-to-the-leading-edge ice shape in order to create some of the curvature observed in the actual scallops as can be seen in Fig. 4.

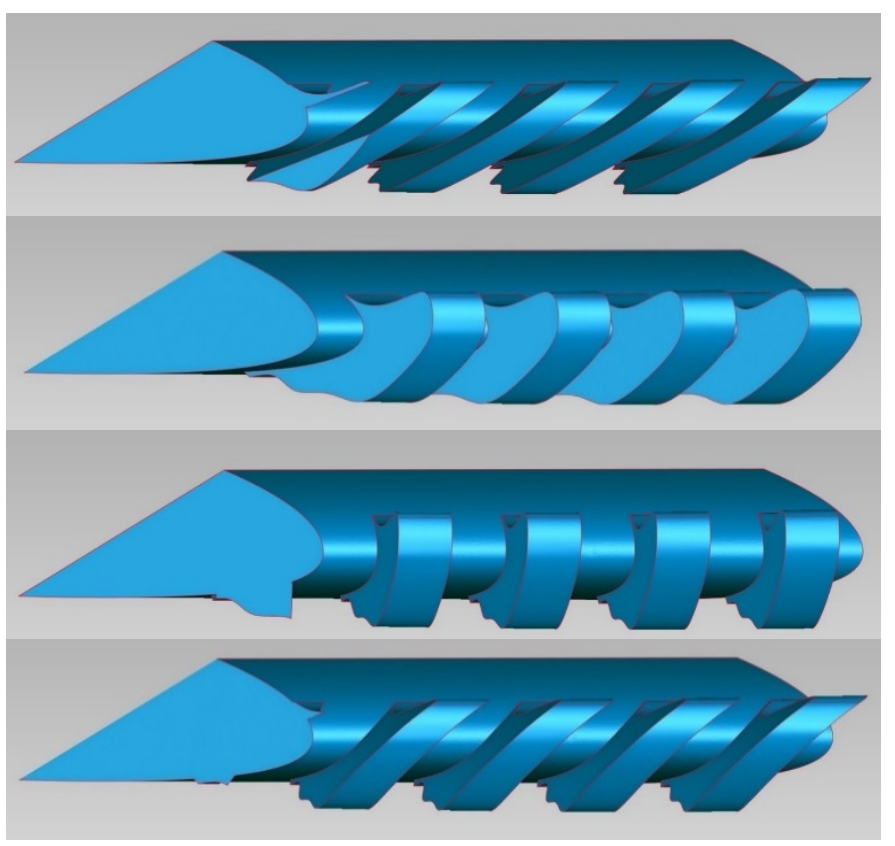

Figure 11. Models of artificial scallop ice shapes. From top to bottom, the ice shapes are: Medium Gap Artificial Scallop, Streamwise Artificial Scallop, Normal-to-Leading-Edge Artificial Scallop, and Curved Artificial Scallop.

\section{III.Results and Discussion}

The goal of this paper is to summarize the aerodynamic-performance differences between various fidelity representations of a scallop ice shape. The effects of Reynolds and Mach number on both the clean and iced wing were discussed in detail by Broeren et al.[23]. The most relevant conclusions from that paper involve the general trends observed in both the clean- and iced-wing performance parameters. For the clean wing, the lift-based parameters are substantially impacted by both Reynolds and Mach number effects. However, drag-based parameters do not show the same strong dependence even for the clean configuration. No Mach number effects were observed, and only weak Reynolds number effects were evident. For the iced configuration, the lift-based parameters showed little to no dependence on Reynolds number and Mach number for Mach numbers above 0.18 . The drag-based parameters indicated no Mach number influence and a small reduction in drag coefficients with increasing Reynolds number. A combination of data are shown in this section from both the highest Reynolds number tested in the F1 facility, which is the closest to flight conditions, and the lower Reynolds number WSU facility. Lee et al.[24] described the details of the comparison between the two models tested in two different facilities.

\section{A. Effect of 3D Artificial Ice Shapes on Wing Performance}

As a baseline for subsequent comparisons, the aerodynamic performance data that were previously published by Woodard et al.[25] are presented in Fig. 12 with the clean wing plotted as a reference. This data set comes from the highest Reynolds number case $\left(R e=11.9 \times 10^{6}, M=0.23\right)$ and clearly identifies the substantial

Page 9 of 22 
impact of the artificial ice shapes relative to the clean wing. The most significant impact on performance is readily observed to be the reduction in lift coefficient at high angles of attack and an increase in drag at all angles. For each of the fidelities, the lift coefficient diverges from the linear regime earlier than the clean configuration but continues to increase as the angle of attack increases. In fact, the maximum lift coefficient for the iced configurations occurs at a higher angle of attack than the angle at which the clean wing exhibits a clear stalling behavior identified by the sharp decrease in lift coefficient at slightly less than 16 deg. angle of attack. With a large number of cases for comparison, it is desirable to extract some parameters from these performance plots in order to compare Reynolds and Mach number effects as well as the fidelity variations. As described above using the example of maximum lift coefficient to define stalling angle, the standard metrics of $C_{L, \max }, C_{D \text {,min, and }} \alpha_{\text {stall }}$ might not sufficiently capture the deleterious impact of the ice shapes, especially on lift.
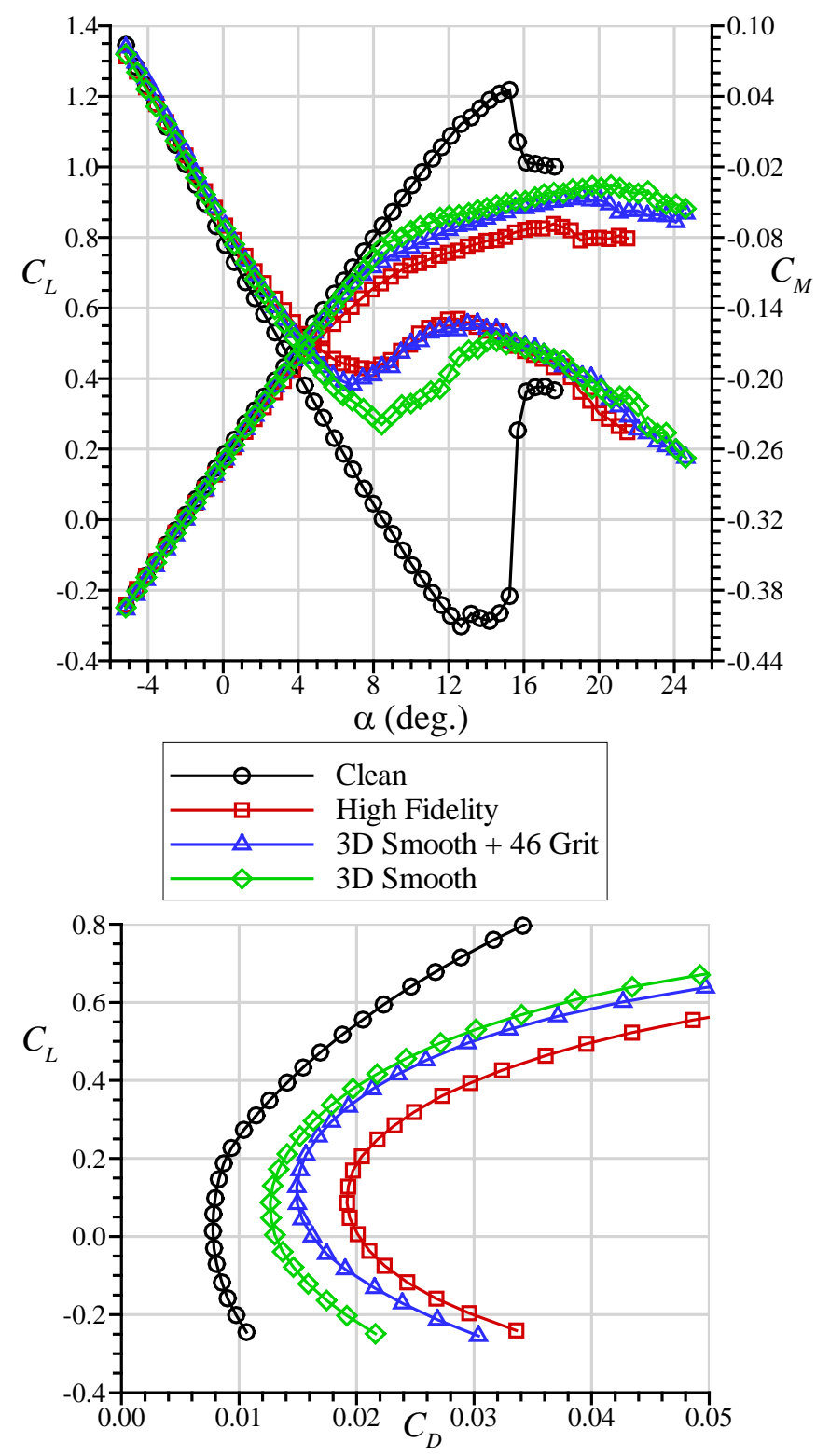

Figure 12. F1 Aerodynamic performance comparison between the three fidelity representations of the Maximum Scallop ice shape at $R e=11.9 \times 10^{6}$, $M=0.23$.

Page 10 of 22
Additional performance parameters have been adapted for application to these swept-wing icing tests. Again, Broeren et al.[23] provide further details of the application to this particular swept wing. In order to more completely capture the stall progression on the swept wing, a performance parameter called "usable" or "inflection" lift is defined based on past work by Furlong and McHugh [39]. This lift coefficient is defined to be coincident with the first local minimum in the pitching moment coefficient calculated about the quarter-chord of the mean aerodynamic chord. As the flow separation progresses inboard from the wing tip, the pitching moment about the quarterchord of the mean aerodynamic chord eventually begins to increase indicating a substantial flow separation. As the data in Fig. 12 (and subsequent figures) illustrate, the first local minimum in the pitchingmoment coefficient nearly corresponds to the angle of attack where the lift coefficient diverges from the linear range. These lift parameters are specifically denoted as $C_{L, \text { use }}$ and $\alpha_{\text {use }}$.

A useful additional parameter can also be extracted from the drag data. The minimum drag value is a convenient parameter for comparing the ice-shape fidelity variations since it occurs near zero lift, so the influence of induced drag is minimized. However, an aircraft does not operate at zero lift, so another point of comparison, at higher lift coefficient, is desirable. Lynch and Khodadoust [40] suggest a process for determining this drag coefficient, and Broeren et al.[23] explain the implementation for this particular swept wing. A lift coefficient equal to 0.6 was identified as the reference value for determining the drag coefficient, and this associated drag value is denoted as $C_{D, 0.6}$ to indicate that it is the drag coefficient when $C_{L}=$ 0.6 . All of the aerodynamic performance data from the F1 tests were analyzed to determine these parameters, and selected cases from the WSU tests were evaluated as well.

\section{B. Wing Performance Effects due to Roughness on an Artificial Ice Shape}

The fidelity of 3D smooth ice shapes can be increased by adding grit roughness to simulate the roughness associated with an actual ice accretion. Previous studies, like the one shown in Fig. 12, simply involved adding grit of a single size to the ice shape. At F1 scale, this standard grit size corresponds to 46 grit as described in Section II.C. A comparison was performed between the performance of the 3D smooth ice shape with 46 grit and with patterned hemispheres of the same height. Figure 13 shows that the performance effects due to the grit and the hemispheres are nearly identical. The high fidelity and 3D smooth ice shapes are included for comparison in the figure. The impact of the roughness relative to the $3 \mathrm{D}$ smooth ice shape is more obvious at extremely high angles of attack in lift performance and in the drag performance over the entire angle of attack range. The roughness increases the drag slightly relative to the smooth ice shape, but the type of roughness (grit versus hemispheres) is inconsequential. The primary difference between the two methods for generating the roughness is the density of the roughness elements, and these data suggest that the density is not critical for this ice shape. The sensitivity to the size of the roughness was also investigated, and the data are presented in Fig. 14. The high fidelity data are shown for comparison, and the 46-grit case is considered the baseline grit size. The drag data illustrate the expected trend with the drag increasing with increasing roughness element size over the entire range of angles of attack tested. The difference in drag between the smallest size ( 80 grit) and the baseline size are small for all angles of attack. The largest size (20 grit) results in a more substantial increase in drag although the drag is still lower than the high fidelity configuration. The effects on the lift performance were more subtle. In the usable lift range, the impact of the roughness size 
was extremely small, but beyond $\alpha_{u s e}$ the smallest grit size resulted in a marginally higher lift coefficient. For this ice shape tested at this Reynolds number, the impact of the roughness element size did not have a significant impact on the lift.
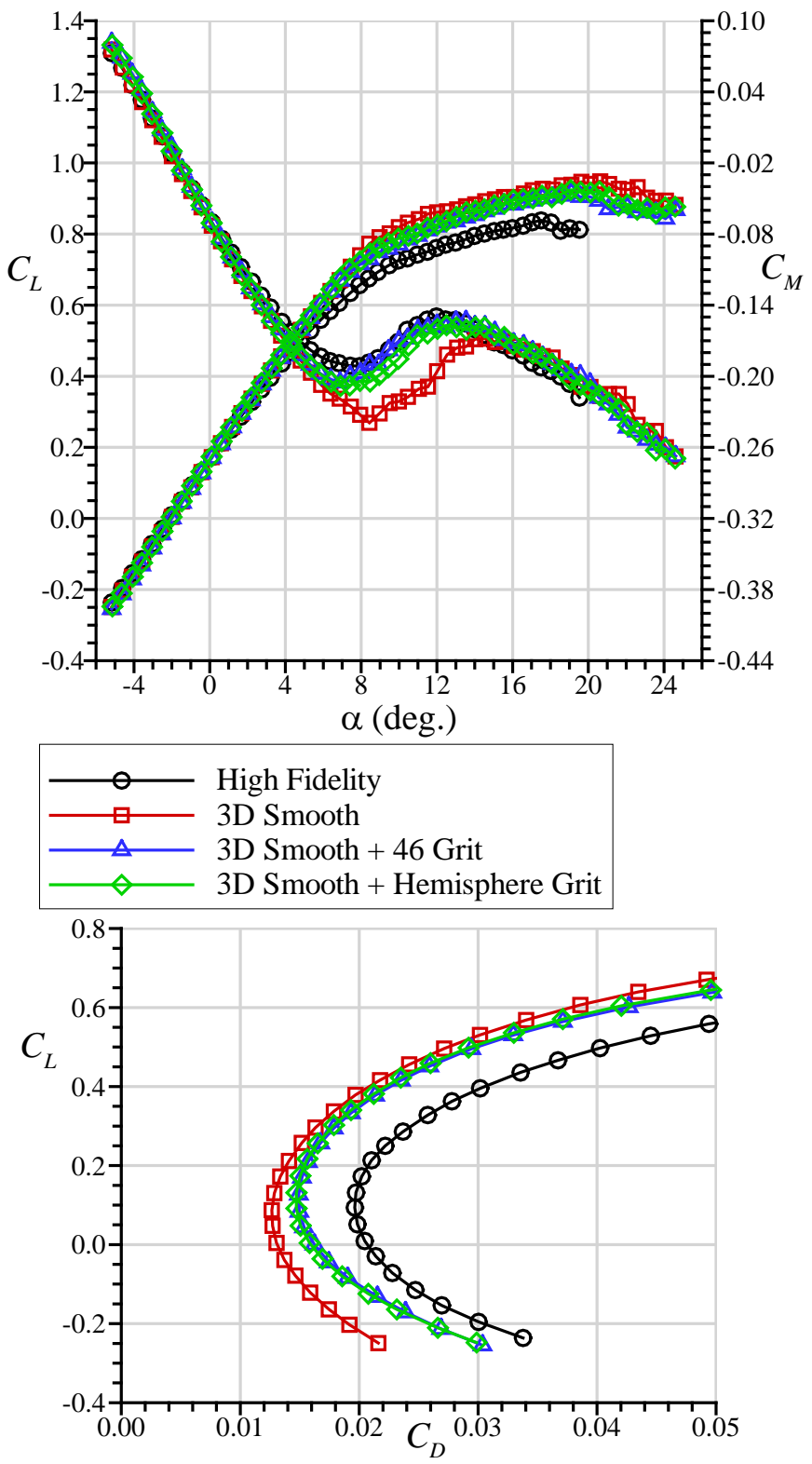

Figure 13. F1 Aerodynamic performance comparison between grit roughness and hemisphere roughness on the 3D Smooth version of the Maximum Scallop ice shape at $R e=11.8 \times 10^{6}, M=0.23$. The height of the grit and the hemispheres is approximately the same.
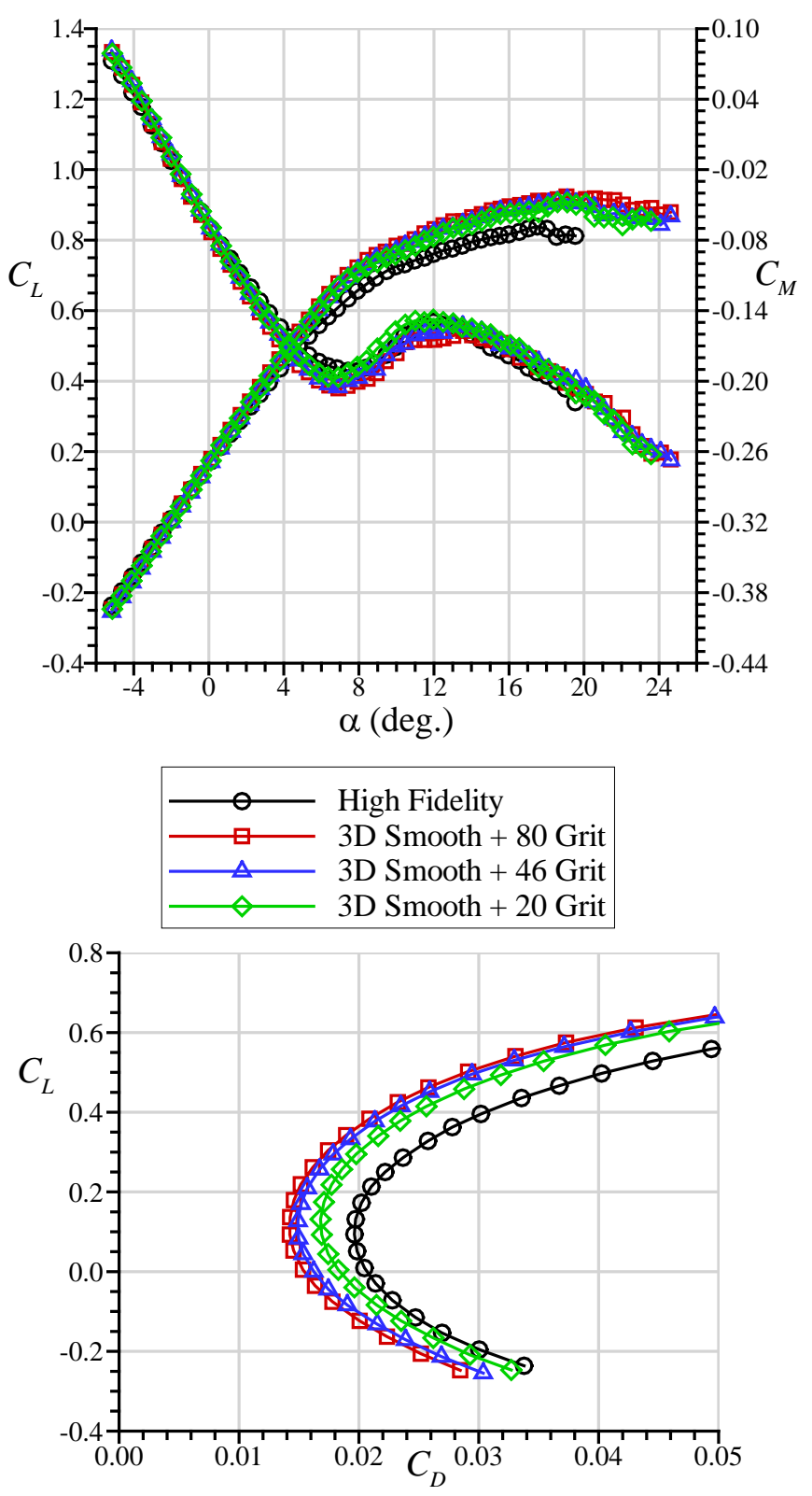

Figure 14. F1 Aerodynamic performance comparison between the three grit sizes on the 3D Smooth version of the Maximum Scallop ice shape at $R e=$ $11.8 \times 10^{6}, M=0.23$.

The lift, angle of attack, and drag data are tabulated for this roughness size study in Tables $4-6$ for the same Reynolds number shown in Fig. 13. The data from the 3D smooth ice shape is included in the table for comparison. The differences and percent differences are tabulated relative to the high fidelity ice shape using the formulas given in the table. The positive values in the lift coefficient difference table indicate that the lift was higher than the high fidelity configuration for all of the roughness study cases, and the negative values in the drag coefficient difference tables indicate that the drag was lower than the high fidelity configuration for these cases.

Further, as Table 5 shows, the stall angle of attack was about 1.5 deg. higher for each of the roughness cases relative to the high fidelity, but the useable angle of attack was reduced by 1 deg. The addition of any roughness to the 3D smooth ice shapes improves the comparison to the high fidelity ice shape in all performance parameters. The roughness shape (e.g. grit vs. hemispheres) does not appear to be an 
important driver for $C_{L}, C_{D}$ and $C_{M}$. The size of the roughness resulted in a more notable impact on drag while not having a significant impact on lift-based parameters. Even the largest grit size was approximately $20 \%$ low in $C_{D}$ compared to the high fidelity.

Table 4. Summary of Lift Performance Parameters for Grit Roughness Study Based on 3D Smooth Max Scallop Ice Shape at $R e=11.9 \times 10^{6}, M=0.23$.

\begin{tabular}{|c|c|c|c|}
\hline Ice Shape Fidelity & $C_{L}$ & $\begin{array}{c}\Delta C_{L} \\
\text { Low Fidelity - } \\
\text { High Fidelity }\end{array}$ & $\begin{array}{c}\text { Percent } \Delta C_{L} \\
\text { (Low Fidelity - } \\
\text { High Fidelity) / } \\
\text { High Fidelity }\end{array}$ \\
\hline & \multicolumn{3}{|c|}{$C_{L, \max }$} \\
\hline Clean & 1.218 & - & - \\
\hline 3D High Fidelity & 0.838 & - & - \\
\hline 3D Smooth & 0.947 & 0.109 & $13 \%$ \\
\hline 3D Smooth + 80 Grit & 0.924 & 0.086 & $10 \%$ \\
\hline 3D Smooth + 46 Grit & 0.915 & 0.077 & $9.2 \%$ \\
\hline 3D Smooth + 20 Grit & 0.907 & 0.069 & $8.2 \%$ \\
\hline \multirow[t]{2}{*}{ 3D Smooth + Hem. } & 0.924 & 0.086 & $10 \%$ \\
\hline & \multicolumn{3}{|c|}{$C_{L, \text { use }}$} \\
\hline Clean & 1.122 & - & - \\
\hline 3D High Fidelity & 0.652 & - & - \\
\hline 3D Smooth & 0.773 & 0.120 & $19 \%$ \\
\hline 3D Smooth + 80 Grit & 0.678 & 0.026 & $4.0 \%$ \\
\hline 3D Smooth + 46 Grit & 0.669 & 0.017 & $2.6 \%$ \\
\hline 3D Smooth + 20 Grit & 0.668 & 0.016 & $2.5 \%$ \\
\hline 3D Smooth + Hem. & 0.677 & 0.025 & $3.8 \%$ \\
\hline
\end{tabular}

Table 5. Summary of Angle of Attack Performance Parameters for Grit Roughness Study Based on 3D Smooth Max Scallop Ice Shape at $R e=$ $11.8 \times 10^{6}, M=0.23$.

\begin{tabular}{|c|c|c|c|c|}
\hline & \multicolumn{2}{|r|}{$\alpha_{\text {stall }}$} & \multicolumn{2}{|r|}{$\alpha_{\alpha_{\text {use }}}$} \\
\hline Ice Shape Fidelity & $\begin{array}{c}\alpha_{\text {stall }} \\
\text { deg.) }\end{array}$ & $\begin{array}{c}\Delta \alpha_{\text {stall }} \\
\text { Low Fidelity - } \\
\text { High Fidelity } \\
\text { (deg.) }\end{array}$ & $\begin{array}{c}\alpha_{\text {use }} \\
\text { (deg.) }\end{array}$ & $\begin{array}{c}\Delta \alpha_{\text {use }} \\
\text { Low Fidelity - } \\
\text { High Fidelity } \\
\text { (deg.) }\end{array}$ \\
\hline Clean & 15.2 & - & 12.7 & - \\
\hline 3D High Fidelity & 17.6 & - & 7.9 & - \\
\hline 3D Smooth & 19.6 & 2.1 & 8.4 & 0.5 \\
\hline 3D Smooth +80 Grit & 19.1 & 1.5 & 6.9 & -1.0 \\
\hline 3D Smooth + 46 Grit & 19.1 & 1.5 & 6.9 & -1.0 \\
\hline 3D Smooth +20 Grit & 18.6 & 1.0 & 6.9 & -1.0 \\
\hline 3D Smooth + Hem. & 19.1 & 1.5 & 6.9 & -1.0 \\
\hline
\end{tabular}

Table 6. Summary of Drag Performance Parameters for Grit Roughness Study Based on 3D Smooth Max Scallop Ice Shape at $R e=11.8 \times 10^{6}, M=0.23$.

\begin{tabular}{|c|c|c|c|}
\hline Ice Shape Fidelity & $C_{D}$ & $\begin{array}{c}\Delta C_{D} \\
\text { Low Fidelity - } \\
\text { High Fidelity }\end{array}$ & $\begin{array}{c}\text { Percent } \Delta C_{D} \\
\text { (Low Fidelity - } \\
\text { High Fidelity) / } \\
\text { High Fidelity }\end{array}$ \\
\hline & \multicolumn{3}{|c|}{$C_{D, \min }$} \\
\hline Clean & 0.0078 & - & - \\
\hline 3D High Fidelity & 0.0192 & - & - \\
\hline 3D Smooth & 0.0127 & -0.0065 & $-34 \%$ \\
\hline 3D Smooth + 80 Grit & 0.0142 & -0.0050 & $-26 \%$ \\
\hline 3D Smooth + 46 Grit & 0.0149 & -0.0043 & $-22 \%$ \\
\hline 3D Smooth + 20 Grit & 0.0168 & -0.0024 & $-13 \%$ \\
\hline \multirow[t]{2}{*}{ 3D Smooth + Hem. } & 0.0147 & -0.0045 & $-23 \%$ \\
\hline & \multicolumn{3}{|c|}{$C_{D, 0.6}$} \\
\hline Clean & 0.0226 & - & - \\
\hline 3D High Fidelity & 0.0584 & - & - \\
\hline 3D Smooth & 0.0378 & -0.0206 & $-35 \%$ \\
\hline 3D Smooth + 80 Grit & 0.0412 & -0.0172 & $-30 \%$ \\
\hline 3D Smooth + 46 Grit & 0.0425 & -0.0159 & $-27 \%$ \\
\hline 3D Smooth + 20 Grit & 0.0454 & -0.0130 & $-22 \%$ \\
\hline 3D Smooth + Hem. & 0.0414 & -0.0170 & $-29 \%$ \\
\hline
\end{tabular}

The values in the tables above only represent one Reynolds and Mach number condition. For most cases, this highest Reynolds number is representative, but the data can also be plotted across the entire range of Reynolds numbers as shown in Figs. 15 and 16. The lift and drag coefficient differences from high fidelity are plotted over the range of Reynolds numbers tested for various Mach numbers. Each of the configurations discussed above is plotted with the 3D smooth configuration included for comparison. In lift coefficient, the 3D smooth configuration exhibits some significant Reynolds number dependence, and shows a slight Reynolds number effect in the drag parameters. However, for all of the configurations with any roughness added, essentially no Reynolds number effects are evident. As discussed specifically related to the highest Reynolds number case above, the differences in both lift and drag between the smallest grit size and the baseline size are small. The hemisphere roughness does show some Mach number effects in usable lift coefficient as evident by two offset horizontal lines. The lines connect symbols of the same Mach number. Over the entire range of Reynolds number, the drag parameters both show consistent results with the largest grit size moving the drag values closest to the high fidelity case relative to the other, but still not matching the high fidelity values.
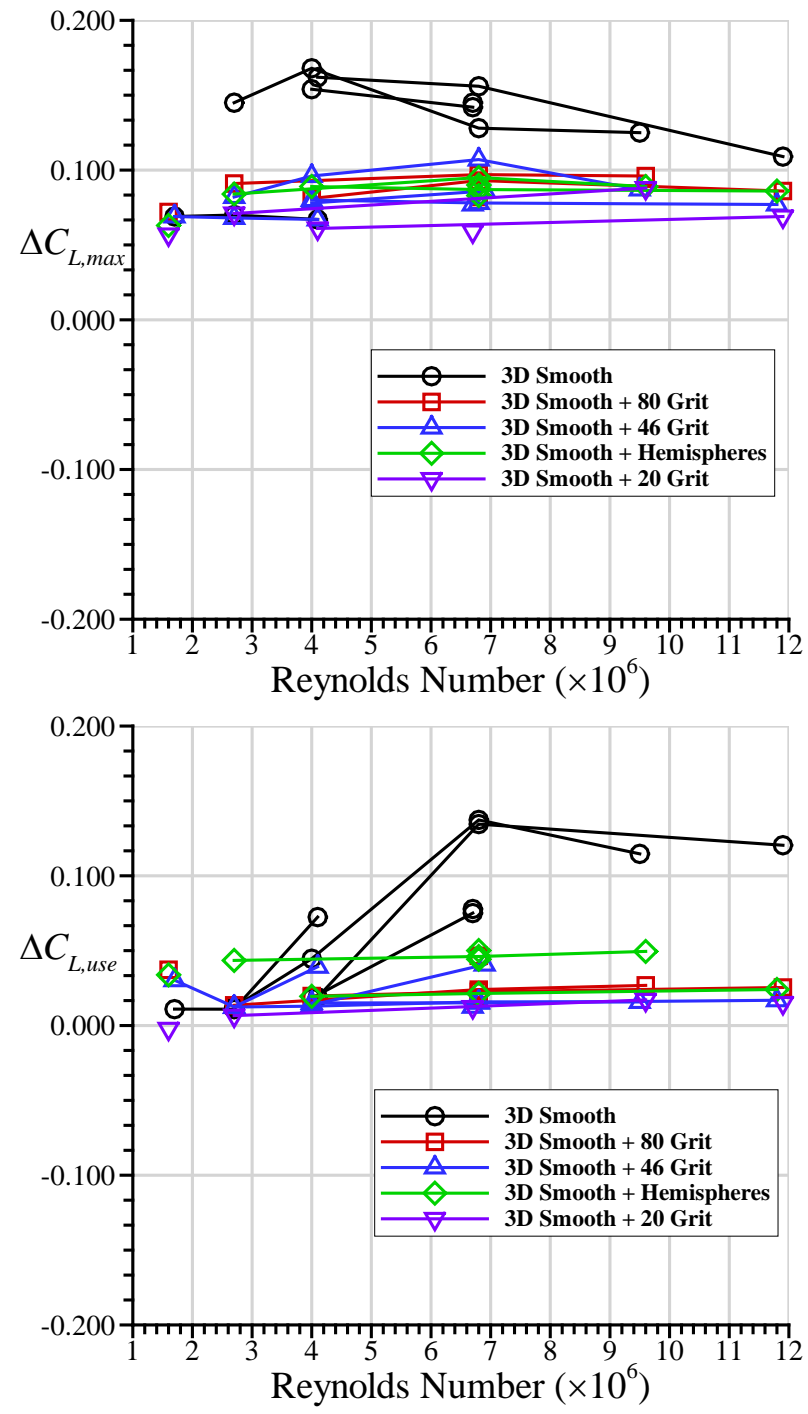

Figure 15. Lift coefficient differences from the high fidelity configuration for various grit roughness sizes plus hemispheres. Mach numbers range from $M$ $=0.09$ to 0.34 . Lines connect symbols with constant Mach number.

Page 12 of 22 

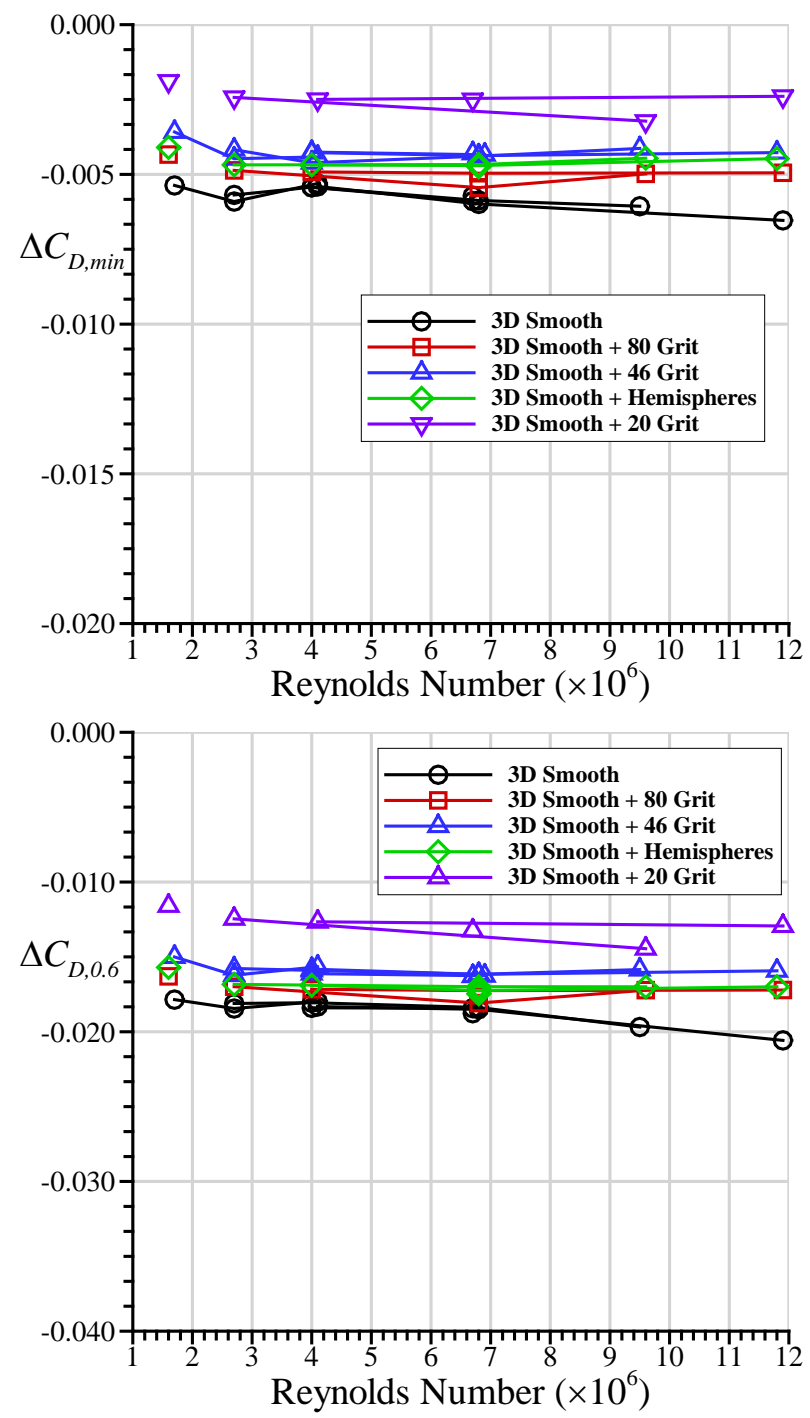

Figure 16. Drag coefficient differences from the high fidelity configuration for various grit roughness sizes plus hemispheres. Mach numbers range from $M$

$=0.09$ to 0.34 . Lines connect symbols with constant Mach number.

\section{Wing performance Effects from LEWICE3D- generated Artificial Ice Shapes}

Ice shapes generated from computer simulation tools serve as another level of fidelity comparison to the experimentally derived ice shapes. The ice shapes created using LEWICE3D in two different ways are described in detail in Section II. C. As described in that section, the LEWICE3D-based ice shapes have significant spanwise variations in the ice-shape parameters, but those variations occur gradually. As such, the computer-generated ice shapes appear similar to the 3D smooth shapes, so the 3D smooth data are included, along with the high fidelity data, for comparisons on the plots in this section. Figure 17 shows the aerodynamic performance data for the LEWICE3D IFB configuration. As expected for an ice shape significantly smaller than the 3D smooth ice shape but with otherwise similar characteristics, the lift is higher, and the drag is lower over the entire tested range. The LEWICE3D IRT ice shape was not tested in this configuration without grit roughness.
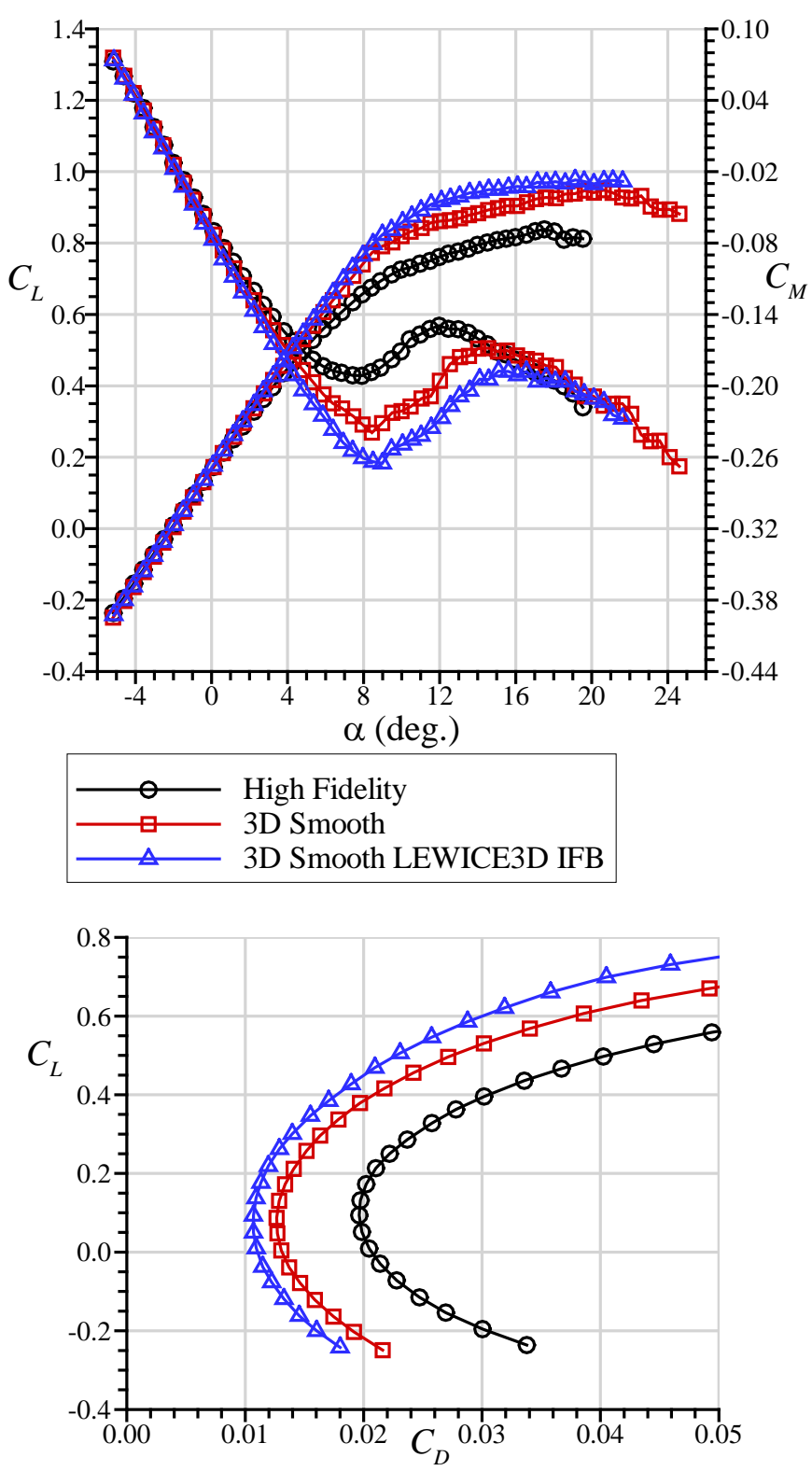

Figure 17. F1 Aerodynamic performance comparison between the 3D smooth version of the maximum scallop ice shape based on the experimental ice accretion and the ice shape generated using LEWICE3D at the Iced Flight Baseline conditions at $R e=11.8 \times 10^{6}, M=0.23$.

The performance data for the two LEWICE3D-based ice shapes with grit roughness added to them are depicted in Fig. 18. The addition of the grit provides a more realistic ice shape as LEWICE3D is not capable of capturing that level of detail. Additionally, computergenerated ice shapes are tested with grit roughness during certification procedures. As Fig. 18 shows, the performance between the two LEWICE3D-based ice shapes is similar at low angles of attack in both lift and drag. At higher angles, some small differences are evident. For example, a small difference in lift between the LEWICE3D ice shapes in the range of 8 to $12 \mathrm{deg}$. angle of attack corresponds to a significant difference in pitching moment suggesting that the IFB ice shape stalls more rapidly than the IRT ice shape. That analysis is consistent with the lower horn angle on the outboard portion of the wing for the IRT ice shape relative to the IFB ice shape. Both the IFB and IRT ice shapes result in higher lift and lower drag on the wing relative to both the 3D smooth with grit ice 
shape and the high fidelity version. All of the performance parameters are tabulated for lift, angle of attack, and drag for this highest Reynolds number and are compared to the high fidelity ice shape in Tables 7 -9. Overall, the performance between the two LEWICE3D-based ice shapes is similar especially when compared relative to the high fidelity ice shape. Both computer generated ice shapes with grit capture approximately $65 \%$ of the high fidelity $C_{D \text {,min }}$ and only approximately $50 \% C_{D, 0.6}$ value. The $3 \mathrm{D}$ smooth plus grit ice shape discussed in the previous section resulted in approximately $75 \%$ of the high fidelity drag effect. Furthermore, the LEWICE3Dbased ice shapes, even with grit, resulted in lift coefficients $14 \%$ to $22 \%$ greater than the high fidelity case compared to the 3D smooth with grit cases that were $3 \%$ to $9 \%$ higher.
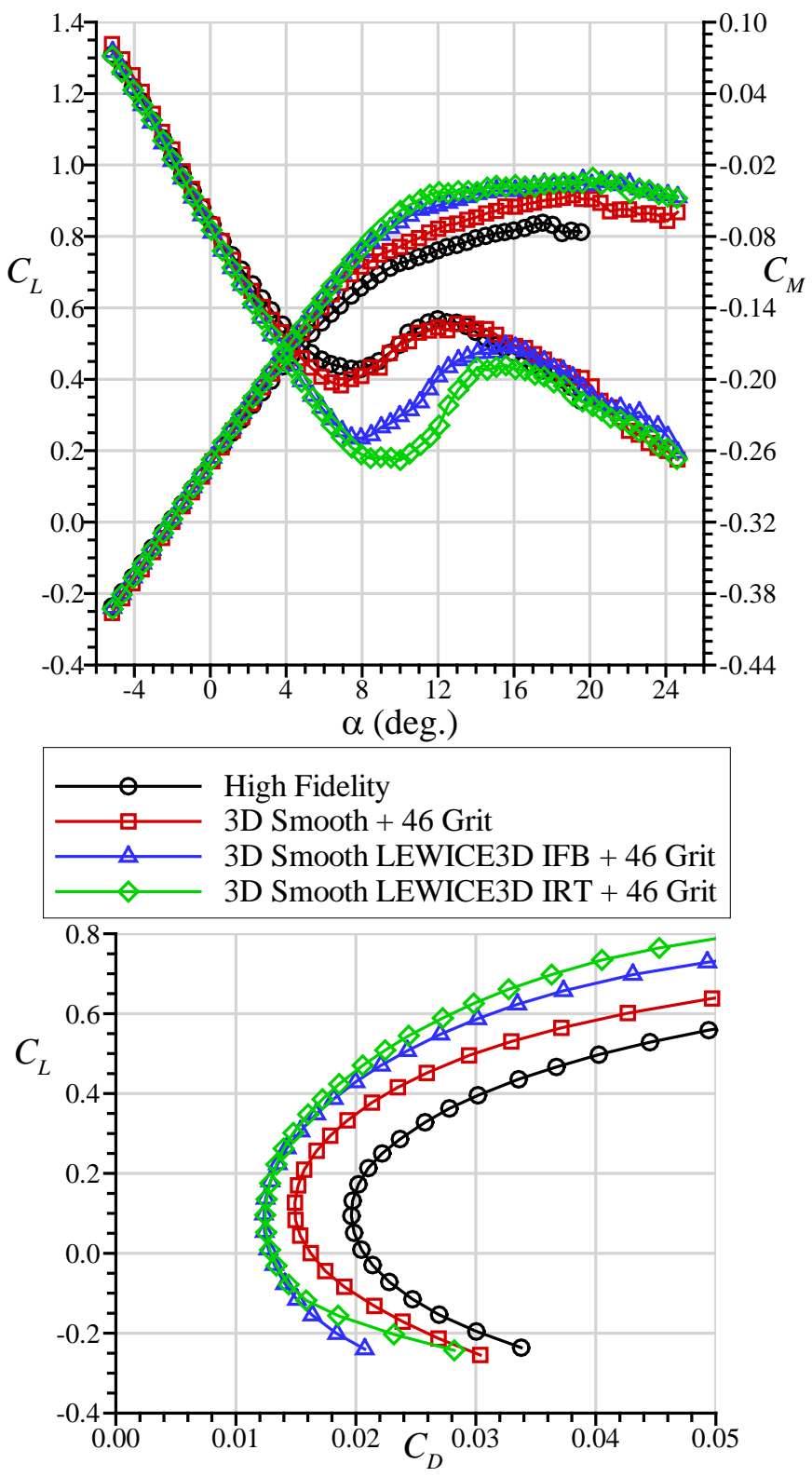

Figure 18. F1 Aerodynamic performance comparison between the 3D smooth version of the maximum scallop ice shape based on the experimental ice accretion and the ice shapes generated using LEWICE3D at both the Iced Flight Baseline conditions and IRT conditions. All ice shapes were tested with grit roughness added at $R e=11.8 \times 10^{6}, M=0.23$.
Table 7. Summary of Lift Performance Parameters for Various Maximum Scallop Condition LEWICE3D-Based Ice Shapes at $R e=11.8 \times 10^{6}, M=0.23$.

\begin{tabular}{|c|c|c|c|}
\hline Ice Shape Fidelity & $C_{L}$ & $\begin{array}{l}\qquad \Delta C_{L} \\
\text { Low Fidelity - } \\
\text { High Fidelity }\end{array}$ & $\begin{array}{c}\text { Percent } \Delta C_{L} \\
\text { (Low Fidelity - } \\
\text { High Fidelity) / } \\
\text { High Fidelity }\end{array}$ \\
\hline & \multicolumn{3}{|c|}{$C_{L, \max }$} \\
\hline Clean & 1.218 & - & - \\
\hline 3D High Fidelity & 0.838 & - & - \\
\hline 3D Smooth & 0.947 & 0.109 & $13 \%$ \\
\hline 3D Smooth + Grit & 0.915 & 0.077 & $9.2 \%$ \\
\hline LEWICE3D IFB & 0.977 & 0.139 & $17 \%$ \\
\hline LEWICE3D IFB + Grit & 0.959 & 0.121 & $14 \%$ \\
\hline \multirow[t]{2}{*}{ LEWICE3D IRT + Grit } & 0.955 & 0.117 & $14 \%$ \\
\hline & \multicolumn{3}{|c|}{$C_{L, \text { use }}$} \\
\hline Clean & 1.122 & - & - \\
\hline 3D High Fidelity & 0.652 & - & - \\
\hline 3D Smooth & 0.773 & 0.120 & $19 \%$ \\
\hline 3D Smooth + Grit & 0.669 & 0.017 & $2.6 \%$ \\
\hline LEWICE3D IFB & 0.823 & 0.171 & $26 \%$ \\
\hline LEWICE3D IFB + Grit & 0.757 & 0.105 & $16 \%$ \\
\hline LEWICE3D IRT + Grit & 0.794 & 0.142 & $22 \%$ \\
\hline
\end{tabular}

Table 8. Summary of Angle of Attack Performance Parameters for Various Maximum Scallop Condition LEWICE3D-Based Ice Shapes at $R e=$ $11.8 \times 10^{6}, M=0.23$.

\begin{tabular}{|c|c|c|c|c|}
\hline & \multicolumn{2}{|r|}{$\alpha_{\text {stall }}$} & \multicolumn{2}{|r|}{$\alpha_{\text {use }}$} \\
\hline Ice Shape Fidelity & $\begin{array}{c}\alpha_{\text {stall }} \\
\text { (deg.) }\end{array}$ & $\begin{array}{c}\Delta \alpha_{\text {stall }} \\
\text { Low Fidelity } \\
- \\
\text { High Fidelity } \\
\text { (deg.) }\end{array}$ & $\begin{array}{c}\alpha_{\text {use }} \\
\text { (deg.) }\end{array}$ & $\begin{array}{c}\Delta \alpha_{\text {use }} \\
\text { Low Fidelity } \\
- \\
\text { High Fidelity } \\
\text { (deg.) }\end{array}$ \\
\hline Clean & 15.2 & - & 12.7 & - \\
\hline 3D High Fidelity & 17.6 & - & 7.9 & - \\
\hline 3D Smooth & 19.6 & 2.1 & 8.4 & 0.5 \\
\hline 3D Smooth + Grit & 19.1 & 1.5 & 6.9 & -1.0 \\
\hline LEWICE3D IFB & 19.1 & 1.5 & 8.9 & 1.0 \\
\hline LEWICE3D IFB + Grit & 19.6 & 2.0 & 7.9 & 0.0 \\
\hline LEWICE3D IRT + Grit & 21.1 & 3.5 & 8.5 & 0.5 \\
\hline
\end{tabular}

Table 9. Summary of Drag Performance Parameters for Various Maximum Scallop Condition LEWICE3D-Based Ice Shapes at $R e=11.8 \times 10^{6}, M=0.23$.

\begin{tabular}{|c|c|c|c|}
\hline Ice Shape Fidelity & $C_{D}$ & $\begin{array}{c}\Delta C_{D} \\
\text { Low Fidelity - } \\
\text { High Fidelity }\end{array}$ & $\begin{array}{c}\text { Percent } \Delta C_{D} \\
\text { (Low Fidelity - } \\
\text { High Fidelity) / } \\
\text { High Fidelity }\end{array}$ \\
\hline & \multicolumn{3}{|c|}{$C_{D, \min }$} \\
\hline Clean & 0.0078 & - & - \\
\hline 3D High Fidelity & 0.0192 & - & - \\
\hline 3D Smooth & 0.0127 & -0.0065 & $-34 \%$ \\
\hline 3D Smooth + Grit & 0.0149 & -0.0043 & $-22 \%$ \\
\hline LEWICE3D IFB & 0.0107 & -0.0085 & $-44 \%$ \\
\hline LEWICE3D IFB + Grit & 0.0123 & -0.0069 & $-36 \%$ \\
\hline \multirow[t]{2}{*}{ LEWICE3D IRT + Grit } & 0.0124 & -0.0068 & $-35 \%$ \\
\hline & \multicolumn{3}{|c|}{$C_{D, 0.6}$} \\
\hline Clean & 0.0226 & - & - \\
\hline 3D High Fidelity & 0.0584 & - & - \\
\hline 3D Smooth & 0.0378 & -0.0206 & $-35 \%$ \\
\hline 3D Smooth + Grit & 0.0425 & -0.0159 & $-27 \%$ \\
\hline LEWICE3D IFB & 0.0300 & -0.0284 & $-49 \%$ \\
\hline LEWICE3D IFB + Grit & 0.0313 & -0.0271 & $-46 \%$ \\
\hline LEWICE3D IRT + Grit & 0.0280 & -0.0304 & $-52 \%$ \\
\hline
\end{tabular}


The performance parameter differences are plotted over the entire range of available Reynolds numbers in Figs. 19 and 20. As discussed in the previous section, the addition of the grit roughness significantly reduces the Reynolds number effects. The maximum lift coefficient and both drag-based parameters show little Reynolds dependence. The LEWICE3D-IRT ice shape does show some Reynolds and Mach number variation in usable lift coefficient. The lower horn angle (close to 0 deg.) may result in the flow separation being more sensitive to Reynolds number. The usable lift coefficient, which is defined based on pitching moment inflection point, is the only parameter where significant differences are evident between the two LEWICE3D ice shapes. In minimum drag coefficient, the differences are insignificant, and in the other drag parameter, the differences are small.
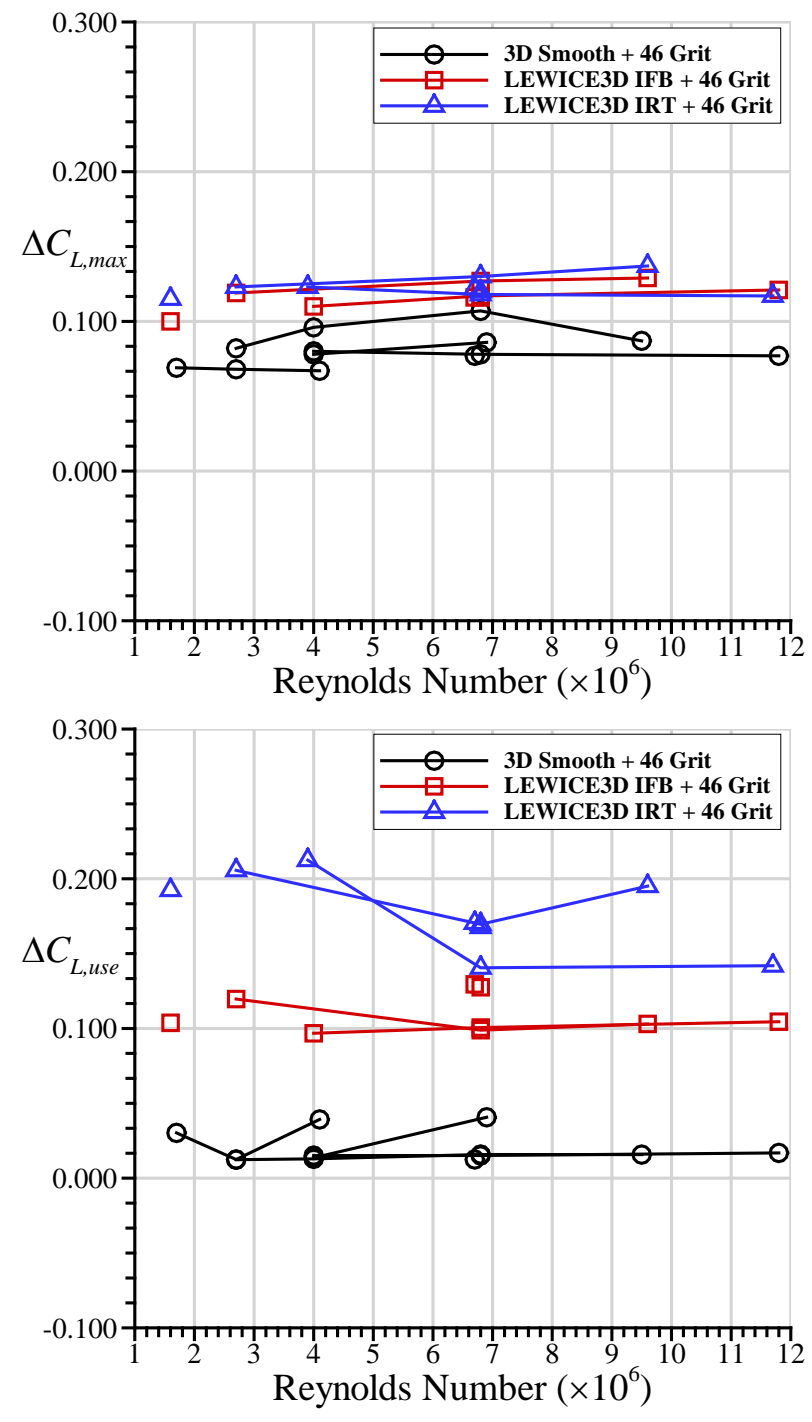

Figure 19. Lift coefficient differences from the high fidelity configuration for two LEWICE3D-generated ice shapes based on the maximum scallop icing conditions. Mach numbers range from $M=0.09$ to 0.34 . Lines connect symbols with constant Mach number.
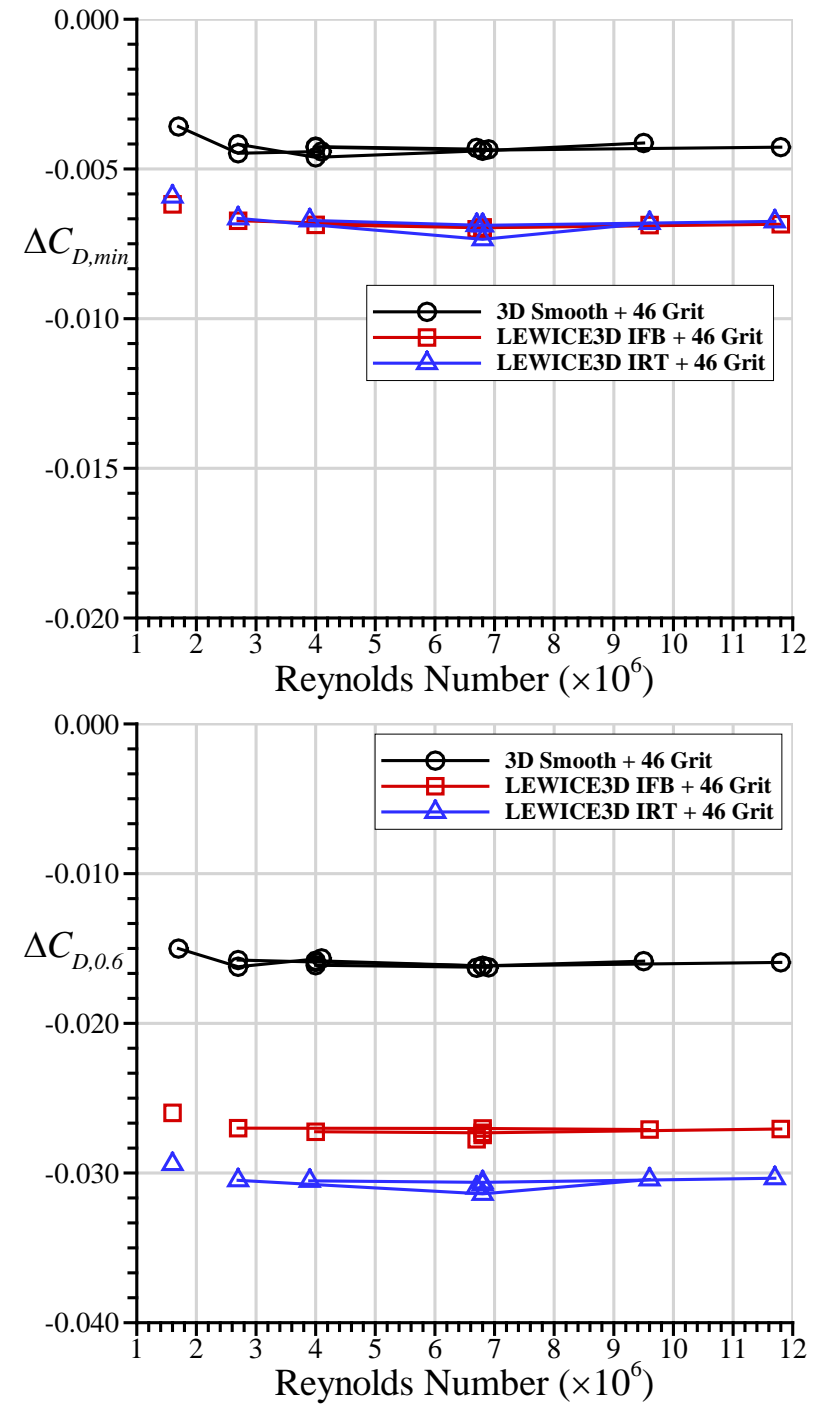

Figure 20. Drag coefficient differences from the high fidelity configuration for two LEWICE3D-generated ice shapes based on the maximum scallop icing conditions. Mach numbers range from $M=0.09$ to 0.34 . Lines connect symbols with constant Mach number.

\section{Wing Performance Effects Due to Spanwise Discontinuities in Artificial Ice Shapes}

Artificial scallop ice shapes were designed to increase the three dimensionality of the 3D smooth ice shapes in an attempt to better understand the impact of the spanwise variations in the high fidelity ice shapes. Two studies were performed by changing different parameters of the artificial scallop ice shapes. The effect of the size of the spanwise gaps cut into the 3D smooth ice shape in order to create three dimensionality was investigated. The highest Reynolds number data from the F1 facility is shown in Fig. $21\left(R e=11.9 \times 10^{6}\right)$, and corresponding data from the low-Reynolds number facility is contained in Fig. $22\left(R e=2.4 \times 10^{6}\right)$. The ice shape with the largest spanwise gaps was only tested on the smaller model. In both figures, the high fidelity and 3D smooth ice-shape data are included, and in Fig. 21, the data from the clean model are included for perspective on the aerodynamic impact of all of these ice shapes. By inspecting the lift performance in both Figs. 21 and 22, the agreement between the artificial scallop and high fidelity ice shapes appears to be good 
especially for the small and medium gap scallop shapes. Looking more closely at the drag and pitching moment, the data indicate some possible differences in the flowfield generated by these ice shapes.
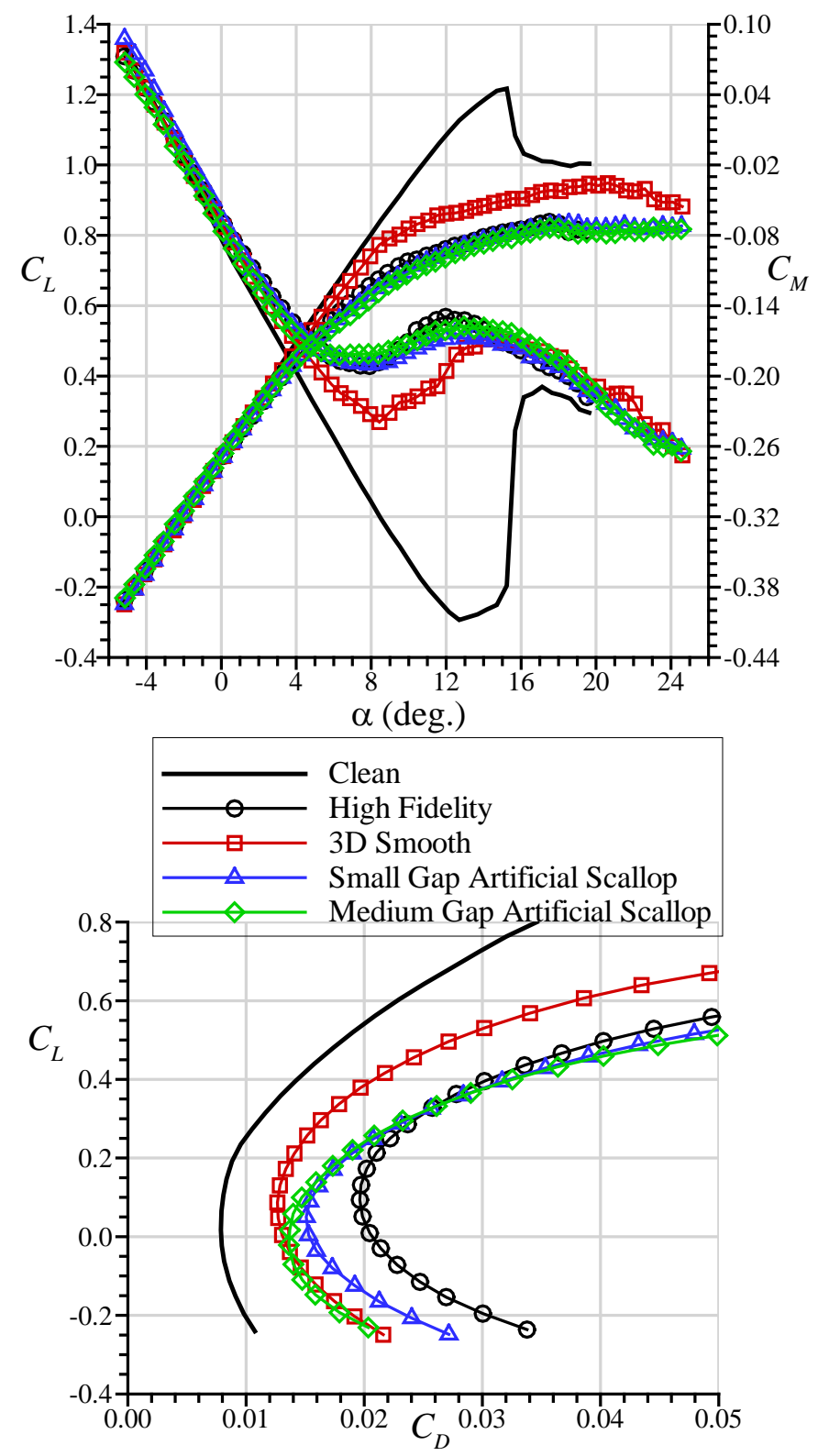

Figure 21. F1 Aerodynamic performance comparison between two artificial scallop ice shapes with varying gap size based on the maximum scallop 3D smooth ice shape at $R e=11.8 \times 10^{6}, M=0.23$.
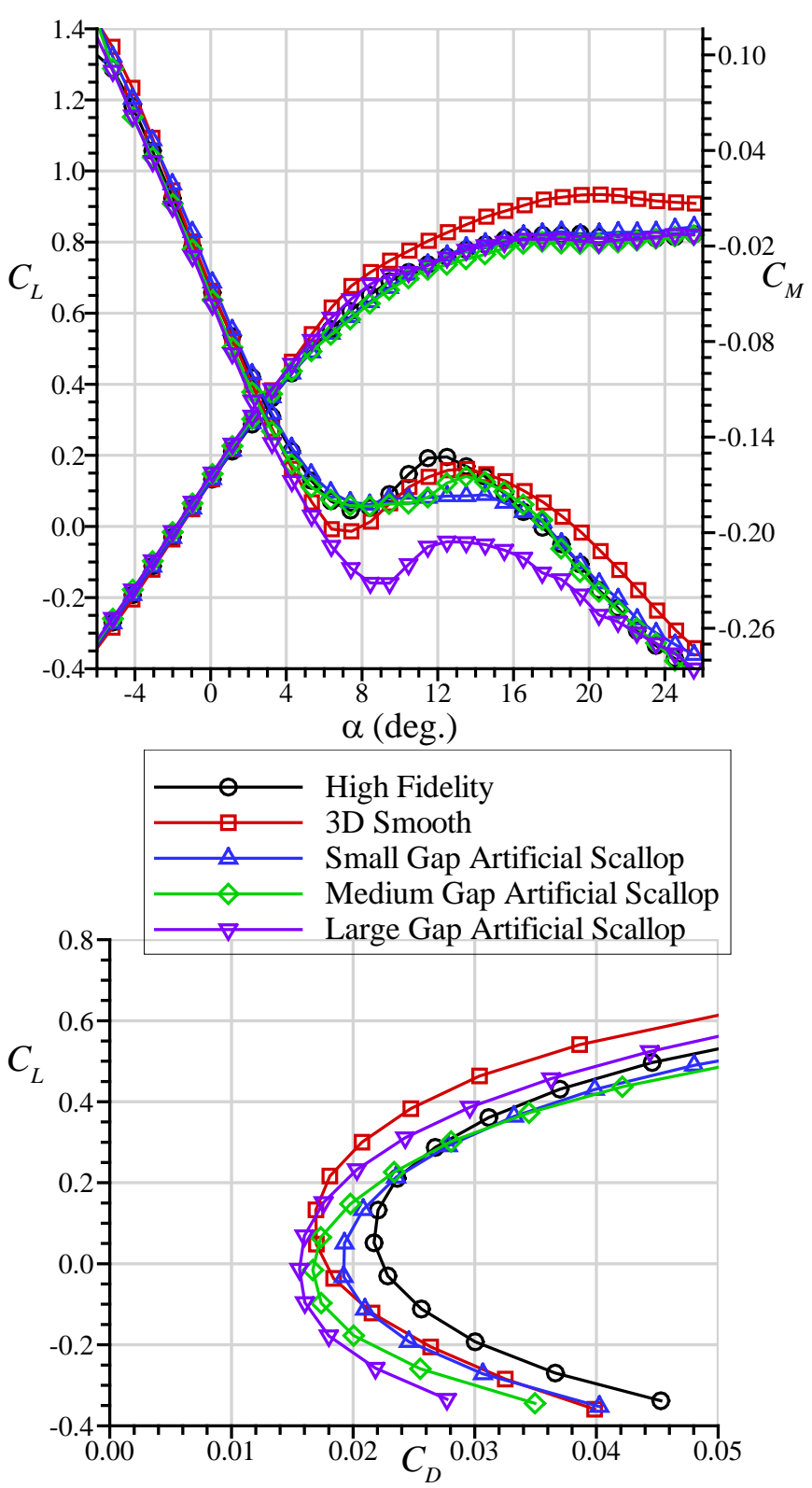

Figure 22. WSU Aerodynamic performance comparison between three artificial scallop ice shapes with varying gap size based on the maximum scallop 3D smooth ice shape at $R e=2.4 \times 10^{6}, M=0.27$.

The performance parameters are contained in Tables 10 - 12 for the artificial scallop ice shapes at the highest Reynolds number. For the small and medium gap scallops, the drag data actually change trends with respect to the high fidelity ice shape. At lower lift coefficients, the high fidelity ice shape has the highest drag, but as the lift increases, the drag from both the small and medium gap ice shapes increases beyond the value from the high fidelity ice shape. For the entire range, the drag from the largest artificial scallop ice shape is lower than all of the other cases. Additionally, the pitching moment data show different trends between the fidelities indicating that the wing may not be stalling in the same way with each of these ice shapes. The usable lift coefficient, which is based on the pitchingmoment inflection point, is calculated to be lower for both the small and medium gap artificial scallop ice shapes relative to the high fidelity shape. Beginning at around 5 deg. angle of attack, the 
performance of the wing is different with these artificial scallop ice shapes relative to the clean wing. Understanding these subtle flowfield differences will require a more detailed investigation into the surface pressures and other flow visualizations that are beyond the scope of the current work.

The other geometric parameter that was studied for these ice shapes involved changing the angle of the gaps cut into the 3D smooth ice shape that create the scallops. The medium gap artificial scallop ice shape was selected as the baseline ice shape for this parametric study. Of the three gap sizes investigated, it had the overall most similar performance to the high fidelity ice shape. Figure 23 illustrates the performance for two scallop-gap orientations with the high fidelity and medium gap artificial scallop.
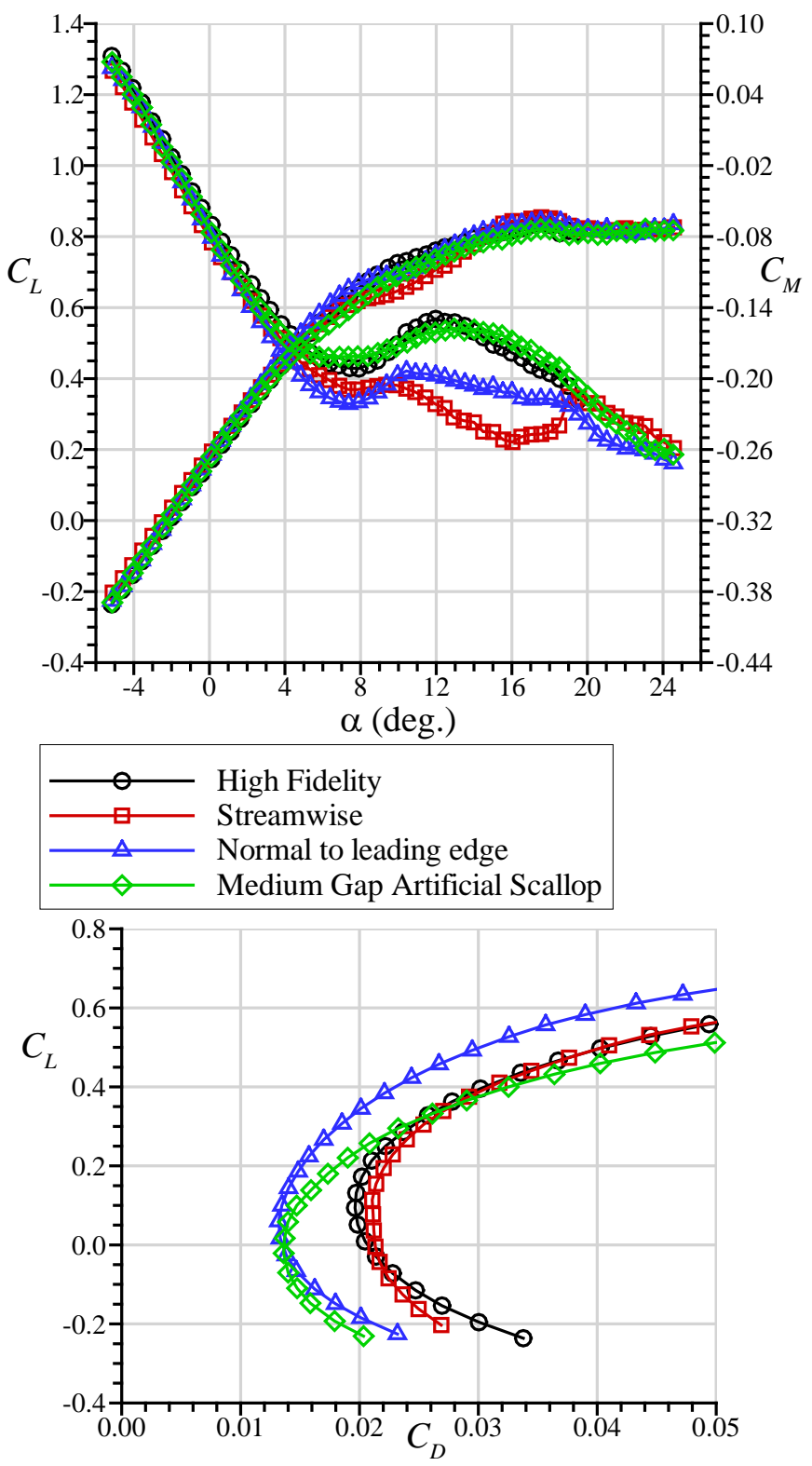

Figure 23. F1 Aerodynamic performance comparison between three artificial scallop ice shapes with varying scallop angle based on the maximum scallop 3D smooth ice shape at $R e=11.8 \times 10^{6}, M=0.23$.
As with the other artificial scallop ice shapes, the overall agreement in the lift data is good between the normal-to-leading-edge scallop and the high fidelity. The behavior of the streamwise scallop through the stalling process is less similar to the other ice shapes. When the wing begins to stall, the lift is reduced more significantly for the streamwise artificial scallop, but it ultimately recovers to a higher lift coefficient than the other artificial scallop ice shapes. In drag, the streamwise artificial scallop results in the best agreement with the high fidelity ice shape. The minimum drag is slightly greater than the high fidelity, but the drag at higher lift coefficients exhibits excellent agreement. The normal-to-the-leading-edge artificial scallop has the worst agreement in drag of any of the artificial scallop ice shapes. The data from the curved artificial scallop are not included in Fig. 23 for clarity. The performance summary is included for that ice shape along with the others in Tables $10-12$. Despite being designed to more closely resemble the appearance of the high fidelity ice shape, the curved artificial scallop showed poor agreement to the high fidelity ice shape in both lift and drag parameters.

Table 10. Summary of Lift Performance Parameters for artificial scallop ice shapes with varying parameters based on the maximum scallop 3D smooth ice shape at $R e=11.8 \times 10^{6}, M=0.23$.

\begin{tabular}{|c|c|c|c|}
\hline Ice Shape Fidelity & $C_{L}$ & $\begin{array}{c}\Delta C_{L} \\
\text { Low Fidelity - } \\
\text { High Fidelity }\end{array}$ & $\begin{array}{c}\text { Percent } \Delta C_{L} \\
\text { (Low Fidelity - } \\
\text { High Fidelity) / } \\
\text { High Fidelity }\end{array}$ \\
\hline & \multicolumn{3}{|c|}{$C_{L, \max }$} \\
\hline Clean & 1.218 & - & - \\
\hline 3D High Fidelity & 0.838 & - & - \\
\hline 3D Smooth & 0.947 & 0.109 & $13 \%$ \\
\hline Small Gap & 0.834 & -0.004 & $-0.5 \%$ \\
\hline Medium Gap & 0.821 & -0.017 & $-2.0 \%$ \\
\hline Streamwise & 0.854 & 0.016 & $1.9 \%$ \\
\hline Normal-to-Leading-Edge & 0.843 & 0.005 & $0.6 \%$ \\
\hline \multirow[t]{2}{*}{ Curved } & 0.779 & -0.059 & $-7.0 \%$ \\
\hline & \multicolumn{3}{|c|}{$C_{L, \text { use }}$} \\
\hline Clean & 1.122 & - & - \\
\hline 3D High Fidelity & 0.652 & - & - \\
\hline 3D Smooth & 0.773 & 0.120 & $19 \%$ \\
\hline Small Gap & 0.629 & -0.023 & $-3.5 \%$ \\
\hline Medium Gap & 0.551 & -0.101 & $-16 \%$ \\
\hline Streamwise & 0.618 & -0.034 & $-5.2 \%$ \\
\hline Normal-to-Leading-Edge & 0.653 & 0.001 & $0.2 \%$ \\
\hline Curved & 0.573 & -0.079 & $-12 \%$ \\
\hline
\end{tabular}

Table 11. Summary of Angle of Attack Performance Parameters for artificial scallop ice shapes with varying parameters based on the maximum scallop 3D smooth ice shape at $R e=11.8 \times 10^{6}, M=0.23$.

\begin{tabular}{|c|c|c|c|c|}
\hline & & $\alpha_{\text {stall }}$ & & $\alpha_{\text {use }}$ \\
\hline Ice Shape Fidelity & $\begin{array}{c}\alpha_{\text {stall }} \\
\text { (deg.) }\end{array}$ & $\begin{array}{c}\Delta \alpha_{\text {stall }} \\
\text { Low Fidelity } \\
- \\
\text { High Fidelity } \\
\text { (deg.) }\end{array}$ & $\begin{array}{c}\alpha_{\text {use }} \\
\text { (deg.) }\end{array}$ & $\begin{array}{c}\Delta \alpha_{\text {use }} \\
\text { Low Fidelity } \\
- \\
\text { High Fidelity } \\
\text { (deg.) }\end{array}$ \\
\hline Clean & 15.2 & - & 12.7 & - \\
\hline 3D High Fidelity & 17.6 & - & 7.9 & - \\
\hline 3D Smooth & 19.6 & 2.1 & 8.4 & 0.5 \\
\hline Small Gap & 18.6 & 0.9 & 7.9 & 0.0 \\
\hline Medium Gap & 17.5 & -0.1 & 6.3 & -1.6 \\
\hline Streamwise & 17.6 & 0.0 & 7.8 & -0.1 \\
\hline Normal-to-Leading-Edge & 17.5 & -0.1 & 7.4 & -0.5 \\
\hline Curved & 18.0 & 0.4 & 7.8 & -0.1 \\
\hline
\end{tabular}


Table 12 Summary of Drag Performance Parameters for artificial scallop ice shapes with varying parameters based on the maximum scallop 3D smooth ice shape at $R e=11.8 \times 10^{6}, M=0.23$.

\begin{tabular}{|c|c|c|c|}
\hline Ice Shape Fidelity & $C_{D}$ & $\begin{array}{l}\qquad \Delta C_{D} \\
\text { Low Fidelity - } \\
\text { High Fidelity }\end{array}$ & $\begin{array}{c}\text { Percent } \Delta C_{D} \\
\text { (Low Fidelity - } \\
\text { High Fidelity) / } \\
\text { High Fidelity }\end{array}$ \\
\hline & \multicolumn{3}{|c|}{$C_{D, \min }$} \\
\hline Clean & 0.0078 & - & - \\
\hline 3D High Fidelity & 0.0192 & - & - \\
\hline 3D Smooth & 0.0127 & -0.0065 & $-34 \%$ \\
\hline Small Gap & 0.0151 & -0.0041 & $-21 \%$ \\
\hline Medium Gap & 0.0136 & -0.0056 & $-29 \%$ \\
\hline Streamwise & 0.0211 & 0.0019 & $9.9 \%$ \\
\hline Normal-to-Leading-Edge & 0.0132 & -0.0060 & $-31 \%$ \\
\hline \multirow[t]{2}{*}{ Curved } & 0.0164 & -0.0028 & $-15 \%$ \\
\hline & \multicolumn{3}{|c|}{$C_{D, 0.6}$} \\
\hline Clean & 0.0226 & - & - \\
\hline 3D High Fidelity & 0.0584 & - & - \\
\hline 3D Smooth & 0.0378 & -0.0206 & $-35 \%$ \\
\hline Small Gap & 0.0693 & 0.0109 & $19 \%$ \\
\hline Medium Gap & 0.0786 & 0.0202 & $35 \%$ \\
\hline Streamwise & 0.0587 & 0.0003 & $0.5 \%$ \\
\hline Normal-to-Leading-Edge & 0.0415 & -0.0169 & $-29 \%$ \\
\hline Curved & 0.1033 & 0.0449 & $77 \%$ \\
\hline
\end{tabular}

The effects of Reynolds and Mach number were also investigated for all of the artificial scallop ice shapes discussed above. Figures 24 and 25 show the performance parameter differences from the high fidelity over the entire range of Reynolds numbers tested. In maximum lift coefficient and both drag parameters, essentially no Reynolds or Mach number effects are indicated. The usable lift coefficient data in Fig. 24 show some spread in the data as a function of both Reynolds and Mach number. However, this result should be carefully interpreted as the usable lift coefficient is extremely sensitive to the definition of the pitching moment inflection point. As an example, the flat portion of the pitching-moment curve shown in Fig. 23 for the streamwise artificial scallop ice shape makes establishing the exact usable lift coefficient challenging. The minimum pitching moment coefficient is especially poorly defined for the curved artificial scallop, so the usable lift coefficient data for that configuration are not included on that plot. More generally, all of the artificial scallop ice shapes (with the exception of the curved one) capture the maximum lift coefficient well. While the drag performance shows no significant Reynolds number effects, the plots in Fig. 25 illustrate the interesting performance of these artificial scallop ice shapes. For example, the normal-to-the-leading edge artificial scallop ice shape exhibits the worst agreement in minimum drag and the best agreement in $C_{D, 0.6}$ relative to the high fidelity ice shape. Additionally, looking at all four of these performance parameters, the streamwise artificial scallop ice shape might appear to best match the high fidelity ice shape. However, looking more closely at the angles of attack near stall (Fig. 23), the performance is significantly different between that ice shape and the high fidelity ice shape indicating different flowfields produced near stall. Future tests may be needed to more fully understand the flowfields downstream of these artificial scallops with spanwise discontinuities. Only a small number of the possible geometric parameters were explored during these tests. A preliminary conclusion is that the apparent geometric similarity between the artificial scallop and the high fidelity ice shapes does not guarantee aerodynamic similarity. These highly three dimensional ice shapes do not appear to have significant Reynolds or Mach number effects which would allow further studies to be performed at relatively low cost testing facilities in order to better understand the complex flowfields.

Page 18 of 22
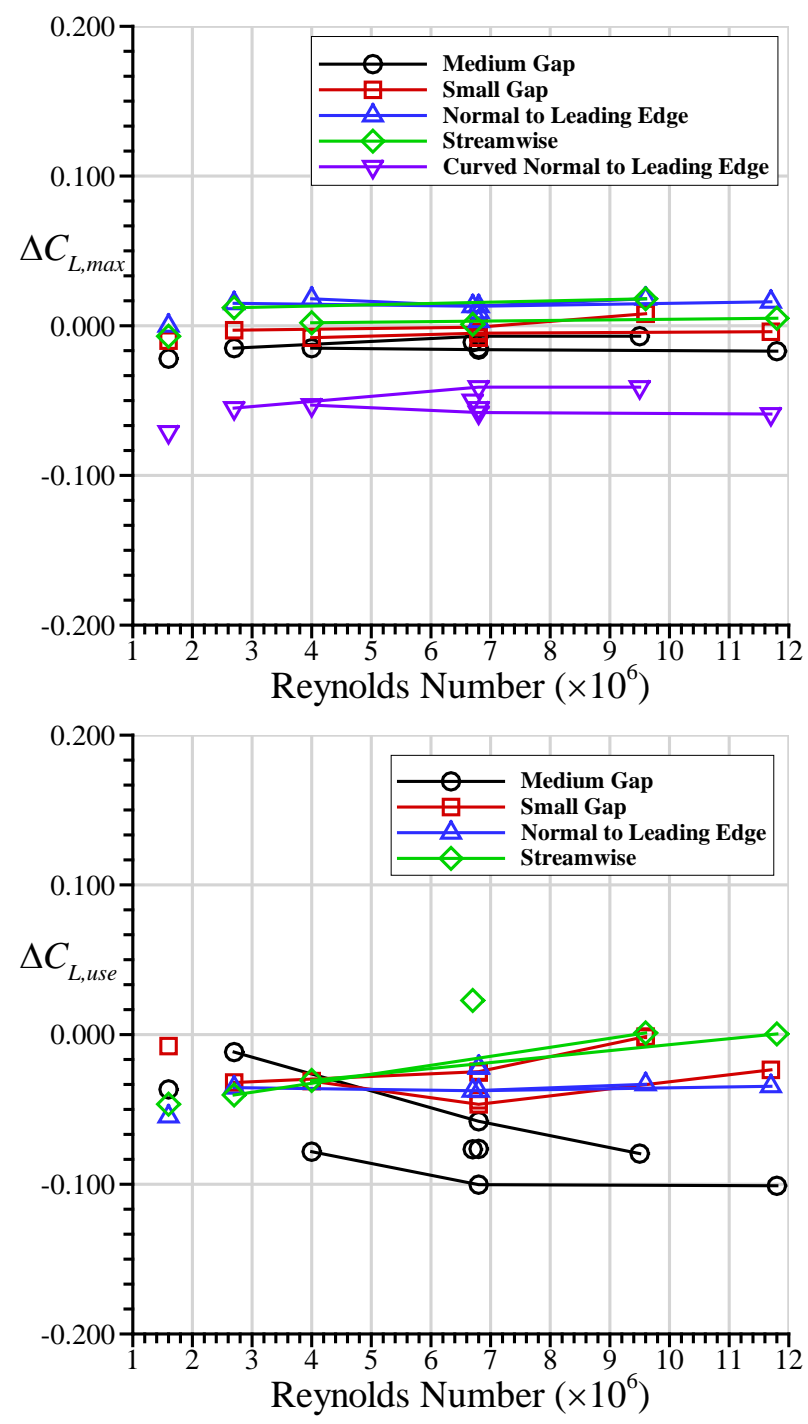

Figure 24. Lift coefficient differences from the high fidelity configuration for the artificial scallop ice shapes based on the maximum scallop icing conditions. Mach numbers range from $M=0.09$ to 0.34 . Lines connect symbols with constant Mach number. 

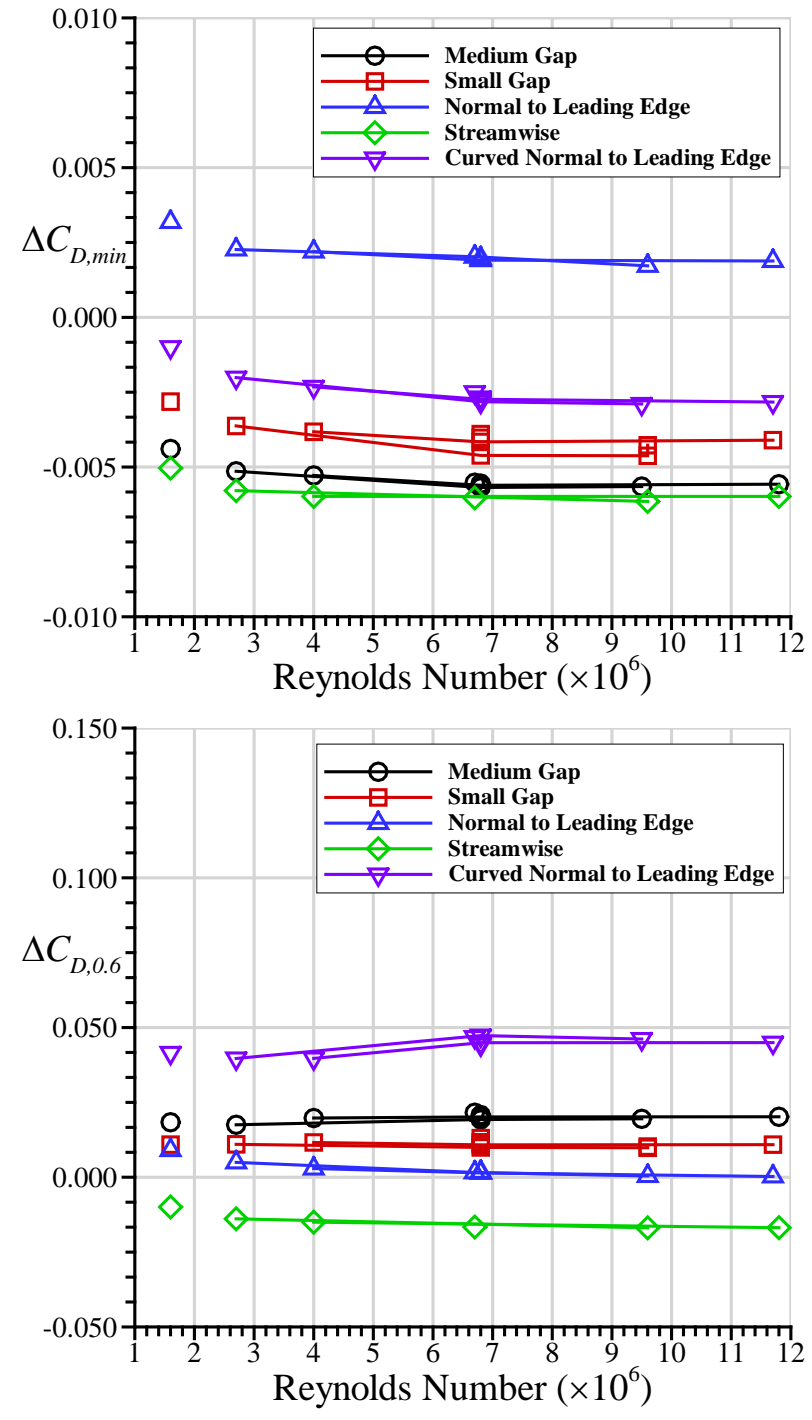

Figure 25. Drag coefficient differences from the high fidelity configuration for the artificial scallop ice shapes based on the maximum scallop icing conditions. Mach numbers range from $M=0.09$ to 0.34 . Lines connect symbols with constant Mach number.

\section{IV.Summary and Conclusion}

This paper primarily presents results from high-Reynolds number aerodynamic testing of a swept wing with various full-span, artificial ice shapes with an emphasis on exploring different representations of one particular ice shape. Additionally, low-Reynolds number aerodynamic data are also presented for comparison to the highReynolds number data for some ice shape configurations which were tested in the two different facilities. The high-Reynolds number tests were conducted at the ONERA F1 wind tunnel using a 13.3\% scale semispan wing model of the CRM65, based on the Common Research Model. The lower-Reynolds number tests were conducted at the WSU Beech wind tunnel using an $8.9 \%$ scale model of the same wing. The two models were geometrical identical except for the scale difference. The model for the F1 wind tunnel is $50 \%$ larger than the model for the WSU wind tunnel. During the wind-tunnel test campaigns, many ice-shape configurations and the clean leadingedge model were tested at a variety of flow conditions, and aerodynamic performance parameters were recorded. Coefficients of lift, drag, and pitching moment along with surface pressures were Page 19 of 22 acquired for angle-of-attack sweeps. The data presented in this work were collected over the range of Reynolds numbers from of $2.7 \times 10^{6}$ to $11.9 \times 10^{6}$ and a Mach number range of 0.18 to 0.34 in the F1 facility and at $R e=1.6 \times 10^{6}, M=0.17$ and $R e=2.4 \times 10^{6}, M=0.26$ in the WSU facility.

The high fidelity ice shape was based on a series of icing wind tunnel tests in the NASA IRT. These tests at full scale provided 3-D laser scans of the ice accretions at three spanwise stations along the wing. Based on those laser scans, full-span ice shapes were developed, and these ice shapes are called "high fidelity" throughout the paper as they capture the highly three-dimensional geometry properties of the ice shapes. Lower fidelity representations can be developed from these full-span, high-fidelity ice shapes. "3D smooth" ice shapes retain the overall spanwise variation in ice-shape size but do not have any of the gaps or scallops along the span. These ice shapes can have grit roughness applied to them to produce another category of ice shapes called "3D smooth + grit." Ice-accretion simulation tools can also be used to produce full-span ice shapes. LEWICE3D-based ice shapes generally appear like 3D smooth ice shapes in that they have gradually or continuously varying shape across the span. The simulations for these ice shapes can be based on a full airplane configuration where the LEWICE3D results are directly scaled to build an ice shape for the wind-tunnel model, or they can simulate IRT tests and be interpolated to develop a full-span ice shape for aerodynamic testing. In an attempt to replicate the highly three dimensional features of the high fidelity ice shapes another ice-shape fidelity can be developed for aerodynamic testing by removing segments from the 3D smooth ice shapes. These ice shapes have spanwise discontinuities similar to the high fidelity ice shapes. Six ice shapes in this category were designed and tested investigating the impact of the size of the gaps between scallop features and the orientation of the cutting plane for the scallops were investigated.

The high fidelity ice shape has a substantial impact on the performance of the wing resulting in reduced lift, reduced stall angle, and increased drag, so performance parameters for other ice shapes are compared to the high fidelity ice shape throughout the work. Past investigations showed that the impact of Reynolds and Mach number was relatively small for the iced configurations, so complete performance data are presented only for the highest Reynolds number cases in this work. In order to better quantify the impact of the iced configurations on the wing, additional performance parameters were applied to the data beyond standard values of maximum lift coefficient and minimum drag coefficient. The parameters are the usable lift coefficient, defined as the lift at the first local minimum in pitching-moment coefficient referenced to the quarter-chord of the mean aerodynamic chord, and the drag coefficient at a specified lift coefficient. For this work, that lift coefficient was selected to be equal to 0.6. These performance parameters are consistent with other swept-wing literature and have also been applied to other studies of this specific data set.

The roughness study on the 3D smooth version of this ice shape indicates that adding any roughness to the smooth ice shapes improves the comparison to the high fidelity ice shape in all parameters. The lift performance is not particularly sensitive to the size of the roughness and the density of the roughness elements. Three grit roughness sizes were tested that spanned a significant size range. The drag performance was most significantly affected by the size of the roughness elements. The larger roughness produced drag results most similar to the high fidelity ice shape. Additionally, the patterned hemisphere roughness, with reduced density relative to the grit, resulted in similar performance to the grit with the same height 
indicating that the shape and style of the elements is considerably less important than the size of the roughness. Generalizing for the highest Reynolds number case, all of the roughness ice shapes over predicted $C_{L, \max }$ and $C_{L \text {,use }}$ by about $10 \%$ and $3 \%$, respectively, relative to the high-fidelity ice shape. The drag penalties associated with all the roughness study cases were all approximately 26\% lower than the high-fidelity ice shape with the exception of the largest grit size that gave a slightly closer result to the high fidelity. Little to no Reynolds and Mach number effects were observed over the tested range for these ice shapes with roughness.

A comparison was performed between LEWICE3D-generated ice shapes and the 3D smooth ice shapes based on the icing experiments. The ice shape resulting from the full airplane simulation was tested without and with grit roughness applied, and the ice shape developed from the simulations of the IRT tests was only tested with grit roughness. Both of the LEWICE3D-based ice shapes are smaller than the experimentally-based ice shape, and as expected, the aerodynamic performance generally follows the size of the ice shape. The lift is increased and drag reduced for the LEWICE3D ice shapes relative to the experimental ice shape. Near stall for the wing, small differences in the performance were evident which are probably due to the differences in the ice horn angle between the two LEWICE3Dbased ice shapes. With roughness applied to these ice shapes, no significant Reynolds number effects were observed.

The study of spanwise discontinuities added to a smooth ice shape in order to recreate the aerodynamic impact of the high fidelity ice shape produced interesting results. Overall, these ice shapes resulted in integrated performance that more closely resembled the high fidelity ice shape than the 3D smooth ice shape (on which they were based). A study of the size of the gaps between the spanwise discontinuities in the ice shapes showed reasonable agreement with the high fidelity data in lift and pitching moment for the small gap and medium gap artificial scallop ice shapes. However, the drag was particularly far off from the high fidelity results at low lift coefficients for those two ice shapes. The angle of the cutting plane for these gaps between the scallops was also varied, and the results require further investigation. The most consistent agreement in drag over the range tested was produced by the streamwise artificial scallop ice shape. However, that ice shape shows poor agreement in lift near stall and is not geometrically similar to the high-fidelity ice shape. The ice shape produced using a curved cutting plane appears most geometrically similar to the high-fidelity ice shape but resulted in poor agreement with the high fidelity lift data. Reynolds and Mach number effects are small for these highly three dimensional ice shapes. Further analysis is needed with the available data in order to better understand the ice shapes with these added spanwise discontinuities. Additional wind tunnel testing could also benefit this understanding by providing the opportunity for more parametric studies. Consistent with past studies, these results indicate that, for highly three-dimensional ice formations like those found on swept wings, the smooth representations are not conservative approximations of the icing impact even when grit roughness is added to the ice shape. Furthermore, simply adding three dimensionality by cutting gaps in the smooth ice shape does not guarantee that the flowfield will match the high-fidelity ice shape.

All of the results presented in this work are based on a single icing condition for a specific swept-wing configuration. More data are necessary in order to generalize these findings to other conditions and configurations.

\section{References}

1. Broeren, A.P., Potapczuk, M.G., Riley, J.T., Villiedieu, P., Moens, F., and Bragg, M.B., "Swept-Wing Ice Accretion Characterization and Aerodynamics,” AIAA Paper 2013-2824, June 2013, also NASA/TM-2013-216555, Sept. 2013.

2. Bragg, M.B., Broeren, A.P., and Blumenthal, L.A., "Iced-Airfoil Aerodynamics,” Progress in Aerospace Sciences, Vol. 41, No. 5, July 2005, pp. 323-418.

3. Broeren, A.P., Diebold, J.M., and Bragg, M.B., "Aerodynamic Classification of Swept-Wing Ice Accretion,” NASA/TM 2013216381, DOT/FAA/TC-13/21, May 2013.

4. Diebold, J.M., Broeren, A.P., and Bragg, M.B., “Aerodynamic Classification of Swept-Wing Ice Accretion,” AIAA Paper 2013-2825, June 2013, also NASA/TM-2013-216556, Sept. 2013.

5. Fujiwara, G.E.C., Bragg, M.B., Camello, S.C., and Lum, C., "Computational and Experimental Ice Accretions of Large Swept Wings in the Icing Research Tunnel,” AIAA Paper 20163734, June 2016.

6. Radenac, E., "Validation of a 3D Ice Accretion Tool on Swept Wing of the SUNSET2 Program," AIAA Paper 2016-3735.

7. Broeren, A.P., Potapczuk, M.G., Lee, S., Malone, A.M., Paul, B.P., Jr., and Woodard, B.S., "Ice-Accretion Test Results for Three Large-Scale Swept-Wing Models in the NASA Icing Research Tunnel,” AIAA Paper 2016-3733, June 2016; also NASA/TM-2016-219137, Sept. 2016.

8. Broeren, A.P., Addy, H.E., Jr., Bragg, M.B., Busch, G.T., Guffond, D., and Montreuil, E., “Aerodynamic Simulation of Ice Accretion on Airfoils,” NASA/TP_2011-216929, June 2011.

9. Broeren, A.P, Addy, H.E., Jr., Lee, S., Monastero, M.C., "Validation of 3-D Ice Accretion Measurement Methodology for Experimental Aerodynamic Simulation,” NASA/TM-2015218724, July 2015.

10. Vassberg, J.C., DeHann, M.A., Rivers, S.M., and Wahls, R.A., "Development of a Common Research Model for Applied CFD Validation Studies,” AIAA Paper 2008-6919, Aug. 2008.

11. Rivers, M.B., and Dittberner, A., "Experimental Investigation of the NASA Common Research Model,” AIAA Paper 2010-4218, June 2010.

12. Rivers, M.B., and Dittberner, A., "Experimental Investigations of the NASA Common Research Model in the NASA Langley National Transonic Facility and the NASA Ames 11-Ft Transonic Wind Tunnel,” AIAA Paper 2011-1126, Jan. 2011.

13. Vassberg, J. C., Tinoco, E. N., Mani, M., Rider, B., Zickuhr, T., Levy, D.W., Brodersen, O., Eisfeld, B., Crippa, S., Wahls, R. A., Morrison, J. H., Mavriplis, D.J., and Murayama, M., "Summary of the Fourth AIAA CFD Drag Prediction Workshop,” AIAA Paper 2010-4547, June 2010.

14. Fujiwara, G. E., Woodard, B. S., Wiberg, B., Mortonson, A. J., and Bragg, M. B., "A Hybrid Airfoil Design Method for Icing Wind Tunnel Tests," Proceedings of the 5th AIAA Atmospheric and Space Environments Conference, AIAA Paper 2013-2826, San Diego, CA, June 2013.

15. Fujiwara, G. E., Wiberg, B. D., Woodard, B., Bragg, M., "3D Swept Hybrid Wing Design Method for Icing Wind Tunnel Tests", Proceedings of the 6th AIAA Atmospheric and Space Environments Conference, AIAA Paper 2014-2616, Atlanta, GA, June 2014. 
16. Fujiwara, G., Bragg, M., Triphahn, C., Wiberg, B., Woodard, B., Loth, E., Malone, A., Paul, B., Pitera, D., Wilcox, P., and Khodadoust, A., "Development of Experimental Icing Simulation Capability for Full-Scale Swept Wings: Hybrid Design Process, Years 1 and 2,” NASA/CR-2017-219573, 2017.

17. Fujiwara, G. E., and Bragg, M. B., "Method for Designing Hybrid Airfoils for Icing Wind-Tunnel Tests," Journal of Aircraft, Vol. 56, No. 1, 2019, pp. 137-149.

18. Fujiwara, G. E., and Bragg, M. B., "Method for Designing Three-Dimensional Swept Hybrid Wings for Icing Wind-Tunnel Tests,” Journal of Aircraft, Vol. 56, No. 2, 2019, pp 730-746.

19. Broeren, A.P., Woodard, B.S., Diebold, J.M., and Moens, F., "Low-Reynolds Number Aerodynamics of an $8.9 \%$ Scale Semispan Swept Wing for Assessment of Icing Effects,” AIAA Paper 2017-4372; also NASA/TM-2017-219533, July 2017.

20. Camello, S.C., Bragg, M.B., Broeren, A.P., Lum, C.W., Woodard, B.S., and Lee, S., "Effect of Ice Shape Fidelity on Swept-Wing Aerodynamic Performance,” AIAA Paper 20174373, June 2017.

21. Lum, C.W., Sandhu, N., Diebold, J.M., Woodard, B.S., and Bragg, M.B., "The Application of a Five-Hole Probe WakeSurvey Technigue to the Study of Swept Wing Icing Aerodynamics,” AIAA Paper 2017-4374, June 2017.

22. Sandhu, N., Soltani, M.R., Bragg, M.B., Lum, C.W., Woodard, B.S., Broeren, A.P., and Lee, S., "Effect of Simulated Scalloped Ice on the Aerodynamics of a Swept-Wing at Low-Reynolds Number,” AIAA Paper 2018-3495, June 2018.

23. Broeren, A.P., Lee, S., Woodard, B.S., Lum, C.W., Smith, T.G., "Independent Effects of Reynolds and Mach Numbers of the Aerodynamics of an Iced Swept Wing,” AIAA Paper 20183492, June 2018.

24. Lee, S., Broeren, A.P., Woodard, B.S., Lum, C.W., and Smith, T.G., "Comparison of Iced Aerodynamic Measurements on Swept Wing from Two Wind Tunnels,” AIAA Paper 2018-3493, June 2018.

25. Woodard, B.S., Broeren, A.P., Lee, S., Lum, C.W., and Bragg, M.B., "Summary of Ice Shape Geometric Fidelity Studies on an Iced Swept Wing,” AIAA Paper 2018-3494, June 2018.

26. Broeren, A.P., Potapczuk, M.G., Lee, S., Woodard, B.S., Bragg, M.B., and Smith, T.G., "Experimental Aerodynamic Simulation of Glaze Ice Accretion on a Swept Wing,” International Conference on Icing of Aircraft, Engines and Structures, Minneapolis, MN, June 17-21, 2019 (submitted for publication).

27. Lee, S., Broeren, A.P, Woodard, B.S., Lum, C.W., and Smith, T.S., "Comparison of Iced Aerodynamic Measurements on Swept Wing from Two Wind Tunnels, Part II,” International Conference on Icing of Aircraft, Engines and Structures, Minneapolis, MN, June 17-21, 2019 (submitted for publication).

28. Woodard, B.S., Broeren, A.P., Diebold, J.M., and Bragg, M.B., "Preliminary Testing of Low Reynolds Number Aerodynamics for a Swept Wing with Artificial Ice Roughness," DOT/FAA/TC-17/48, Sept. 2017.

29. Barlow, J.B., Rae, W.H., Jr., Pope, A., Low-Speed Wind Tunnel Testing, John Wiley \& Sons, Inc., $3^{\text {rd }}$ Ed., 1999, pp. 367-425.

30. "Data Reduction System: Boundary Corrections Three Dimensional Aircraft,” Walter H. Beech Wind Tunnel Engineering Process Description, April 2014.

31. Addy, H.E., Jr., and Chung, J.J., “A Wind Tunnel Study of Icing Effects on a Natural Laminar Flow Airfoil," AIAA Paper 20000095, Jan. 2000; also NASA/TM-2000-209775, Jan. 2000.

32. Addy, H.E., Jr., Broeren, A.P., Zoeckler, J.G., and Lee, S., “A Wind Tunnel Study of Icing Effects on a Business Jet Airfoil," AIAA Paper 2003-0727, Jan. 2003; also NASA/TM-2003212124, Feb. 2003.

Page 21 of 22
33. Broeren, A.P., Bragg, M.B., and Addy, H.E., Jr., "Effect of Intercycle Ice Accretions on Aerodynamic Performance," Journal of Aircraft, Vol. 41, No. 1, Jan.-Feb. 2004, pp 165-174.

34. Broeren, A.P., Bragg, M.B., Addy, H.E., Jr., Lee, S., Moens, F., and Guffond, D., "Effect of High-Fidelity Ice Accretion Simulations on the Performance of a Full-Scale Airfoil Model," Journal of Aircraft, Vol. 47, No. 1, Jan.-Feb. 2010, pp. 240-254; also NASA/TM-2010-216344, Jun. 2010.

35. Camello, S.C., Lee, S., Lum, C.W., and Bragg, M.B., "Generation of Fullspan Leading-Edge 3D Ice Shapes for Swept-Wing Aerodynamic Testing,” AIAA Paper 2016-3737, June 2016.

36. Federal Aviation Administration, Advisory Circular AC 25-25A, Oct. 27, 2014.

37. Yadlin, Y., Monnig, J.T., Malone, A.M., and Paul, B.P., "Icing Simulation Research Supporting the Ice-Accretion Testing of Large-Scale Swept Wing Models,” NASA/CR—2018-2197891, Mar. 2018, Also DOT/FAA/TC-18/17.

38. Fujiwara, G.E., Bragg, M.B., Camello, S., and Lum, C., "Computational and Experimental Ice Accretions of Large Swept Wings in the Icing Research Tunnel," AIAA Paper 20163734, Washington DC, June 2017.

39. Furlong, G.C., and McHugh, J.G., "A Summary and Analysis of the Low-speed Longitudinal Characteristics of Swept Wings at High Reynolds Number, NACA-TR-1339, Jan. 1957.

40. Lynch, F.T., and Khodadoust, A., "Effects of Ice Accretions on Aircraft Aerodynamics,” Progress in Aerospace Sciences, Vol. 37, No. 8, Nov. 2001, pp. 669-767.

\section{Contact Information}

For questions regarding this paper, please contact Brian Woodard (bswoodrd@illinois.edu).

\section{Acknowledgments}

The authors gratefully acknowledge the assistance of many other individuals and organizations that made this work possible. Specific contributors to conducting these wind tunnel test campaigns and understanding the acquired data were Allery Hsu, Amy Strauch, and Natalie Pfister at the University of Illinois, and Kevin Ho and William Yoshida at the University of Washington. The engineers and technicians at both the ONERA F1 wind tunnel and the WSU Beech wind tunnel were extraordinarily helpful in keeping the tests running smoothly and efficiently. The NASA-supported portion of this research was originally funded under the Atmospheric Environment Safety Technologies Project of the Aviation Safety Program with continued support under the Advanced Air Transport Technology and Aeronautics Evaluation and Test Capabilities Projects of the Advanced Air Vehicles Program. The Universities of Washington and Illinois are funded for this program by FAA grant 15-G-009 with support from Dr. James T. Riley.

\section{Definitions/Abbreviations}

$\begin{array}{ll}C_{D} & \text { drag coefficient } \\ C_{D, \text { min }} & \text { minimum drag coefficient } \\ C_{D, 0.6} & \text { drag coefficient at } C_{L}=0.6\end{array}$


$C_{M}$

$C_{M, \min }$

$C_{p}$

\section{CRM}

CRM65

LWC

M

MAC

MCCS

MVD lift coefficient

maximum lift coefficient

usable lift coefficient

aerodynamic chord pitching

moment

minimum quarter-chord

mean aerodynamic chord

pitching moment

model surface pressure

coefficient

Common Research Model

$65 \%$ scale version of the CRM (full-scale reference for this project)

icing cloud liquid water content

freestream Mach number

mean aerodynamic chord

maximum combined cross

section

median volumetric diameter

of icing cloud drop

distribution
$\operatorname{Re}$

RLE

WSU

$x$

$\Delta C_{D, \min }$

$\Delta C_{L, \max }$

freestream Reynolds number based on mean aerodynamic chord

removable leading edge

Wichita State University

wing streamwise coordinate

wing spanwise coordinate

wing thickness coordinate

model angle of attack

stalling angle of attack, consistent with the maximum lift coefficient

usable angle of attack, consistent with the usable lift coefficient

change in minimum drag coefficient from high fidelity

$\Delta C_{D, 0}$

change in drag coefficient at $C_{L}=0.6$ from high fidelity

change in maximum lift coefficient from high fidelity

$\Delta C_{L, \text { use }}$ change in usable lift coefficient from high fidelity 